Victor Martins Tonso

ESTUDO COMPARATIVO ENTRE AS SEQUÊNCIAS DE DIFUSÃO MONOEXPONENCIAL PADRÃO (FOCUS) E BIEXPONENCIAL (IVIM - INTRAVOXEL INCOHERENT MOTION) NA RESSONÂNCIA MAGNÉTICA DA PRÓSTATA

Dissertação apresentada à Sociedade
Beneficente Israelita Brasileira Albert
Einstein para obtenção do Título de Mestre
em Ciências da Saúde.


Victor Martins Tonso

\title{
ESTUDO COMPARATIVO ENTRE AS SEQUÊNCIAS DE DIFUSÃO MONOEXPONENCIAL PADRÃO (FOCUS) E BIEXPONENCIAL (IVIM - INTRAVOXEL INCOHERENT MOTION) NA RESSONÂNCIA MAGNÉTICA DA PRÓSTATA
}

\author{
Dissertação apresentada à Sociedade \\ Beneficente Israelita Brasileira Albert \\ Einstein para obtenção do Título de Mestre \\ em Ciências da Saúde.
}

Orientador: Prof. Dr. Ronaldo Hueb Baroni 
T666e

Tonso, Victor Martins

Estudo comparativo entre as sequências de difusão monoexponencial padrão (FOCUS) e biexponencial (IVIM - intravoxel incoherent motion) na ressonância magnética da próstata / Victor Martins Tonso. -- São Paulo, 2018. ix, $31 \mathrm{f}$.

Dissertação (Mestrado) - Sociedade Beneficente Israelita Brasileira Albert Einstein. Instituto Israelita de Ensino e Pesquisa Albert Einstein. Programa de PósGraduação em Ciências da Saúde.

Título em inglês: Comparative study between standard monoexponential (FOCUS) and biexponential (IVIM - intravoxel incoherent motion) diffusion sequences in the prostate magnetic resonance.

1. Neoplasias da próstata. 2. Neoplasias urogenitais. 3. Saúde do homem. 4. Imagem por ressonância magnética. 5. Imagem de difusão por ressonância magnética

NLM - WN 185

Elaborada pelo Sistema Einstein Integrado de Bibliotecas 
SOCIEDADE BENEFICENTE ISRAELITA BRASILEIRA ALBERT EINSTEIN

Coordenador do curso de Pós-Graduação: Prof. Dr. Luiz Vicente Rizzo 
Victor Martins Tonso

\section{ESTUDO COMPARATIVO ENTRE AS SEQUÊNCIAS DE DIFUSÃO MONOEXPONENCIAL PADRÃO (FOCUS) E BIEXPONENCIAL (IVIM - INTRAVOXEL INCOHERENT MOTION) NA RESSONÂNCIA MAGNÉTICA DA PRÓSTATA}

Presidente da banca: Prof. Dr. Ronaldo Hueb Baroni

BANCA EXAMINADORA

Membros titulares:

Profa. Dra. Elisa Harumi Kozasa

Prof. Dr. Valdair Francisco Muglia

Prof. Dr. Gustavo Franco Carvalhal

Membros suplentes:

Prof. Dr. Gilberto Szarf

Prof. Dr. Fernando Korkes

Aprovada em: 08/05/2018. 


\section{Dedicatória}

Dedico esta dissertação à minha família, que mesmo longe sempre foi e será a base de tudo, e a todos os colegas do setor de radiologia que de alguma forma contribuíram para este trabalho desde o primeiro dia em que eu cheguei aqui no Einstein. 


\section{Agradecimentos}

Ao Dr. Ronaldo Hueb Baroni, exemplo admirado de profissional, pela orientação deste trabalho e do caminho que tenho trilhado na Radiologia, acreditando sempre no meu sucesso.

Aos Drs. Fernando Ide Yamauchi e Thais Caldara Mussi Andrade, por toda colaboração dispensada neste projeto e disposição permanente em ajudar.

Aos demais colegas do Departamento de Diagnóstico por Imagem do Hospital Israelita Albert Einstein, pelo incentivo e apoio ao longo desta jornada.

À biomédica, Joyce Prado de Oliveira, por todo o empenho e disposição em ajudar no decorrer do projeto.

Ao engenheiro Eduardo Figueiredo por toda ajuda e sugestões despendidas, e à GE HealthCare do Brasil pela parceria nesse projeto.

Aos biomédicos, enfermeiros, técnicos e administrativos do Departamento de Diagnóstico por Imagem do Hospital Albert Einstein, sempre empenhados em obter os melhores exames.

A Elivane da Silva Victor pelo auxílio no planejamento e análise estatística deste trabalho.

Aos Drs. Elisa Harumi Kozasa, Gilberto Szarf, Leopoldo Alves Ribeiro, Edson Amaro Jr., Atul Kumar Taneja e Jose Roberto Colombo, pela participação e contribuições geradas no exame de qualificação.

Ao Dr. Marcelo Buarque de Gusmão Funari, pela confiança no meu trabalho e exemplo diário de profissionalismo e caráter.

Ao Hospital Israelita Albert Einstein, pela chance de aperfeiçoamento contínuo por meio da promoção do ensino e da pesquisa em centro de excelência.

Aos pacientes envolvidos, vital fonte do saber e aos quais esperamos ter ajudado. 


\section{Sumário}

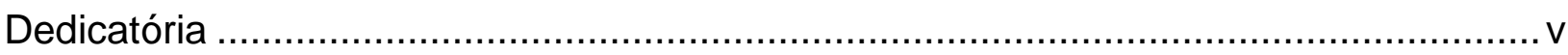

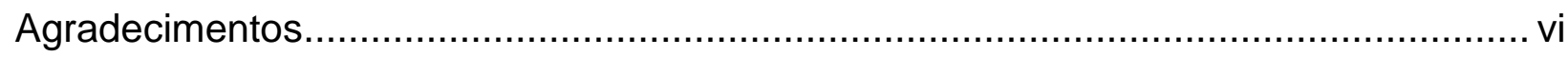

Lista de abreviaturas .................................................................................

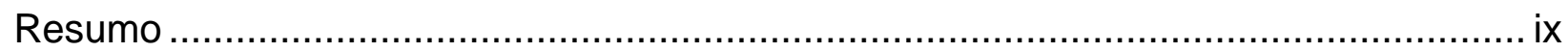

1 INTRODUÇÃO

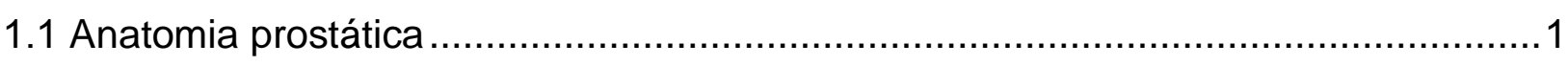

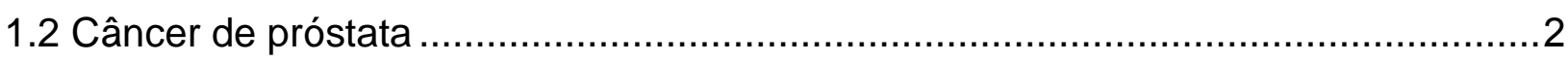

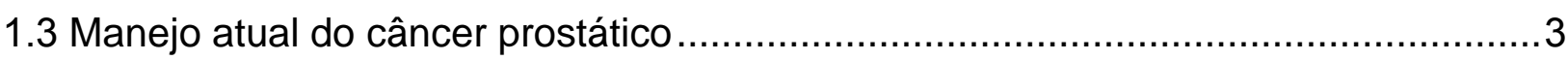

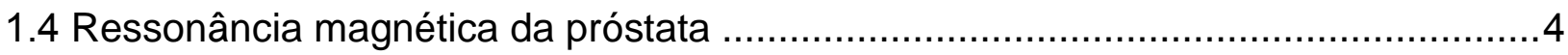

1.5 Difusão na ressonância magnética da próstata ........................................................

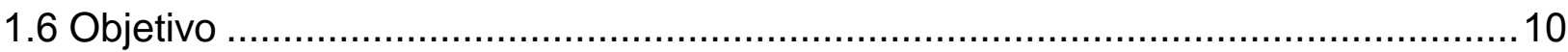

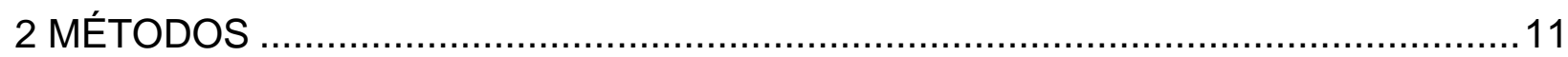

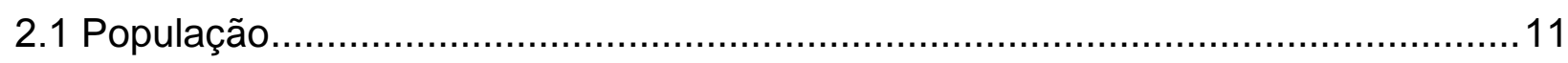

2.2 Protocolo da ressonância magnética..............................................................

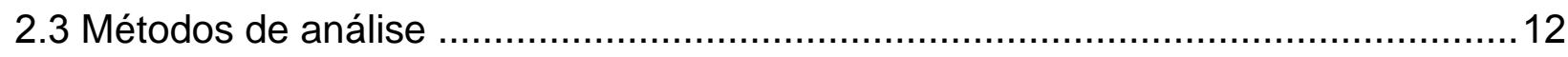

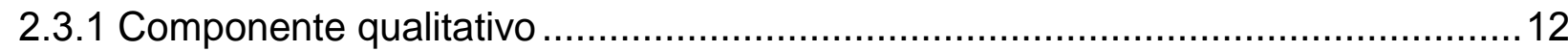

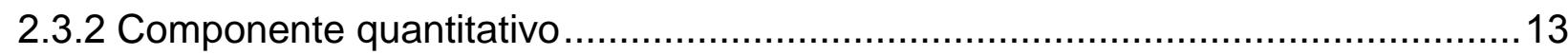

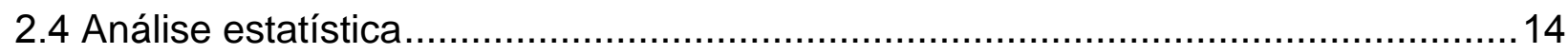

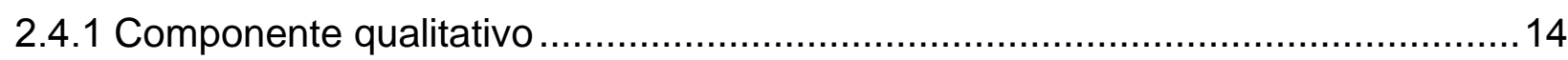

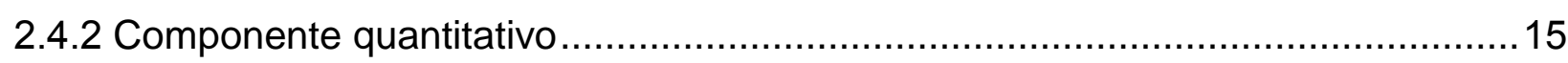

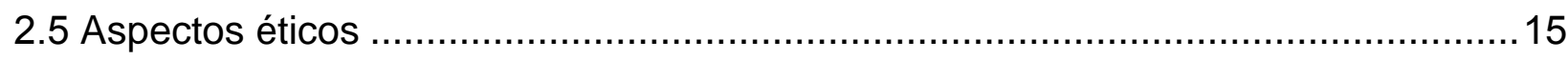

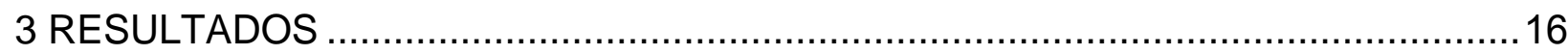

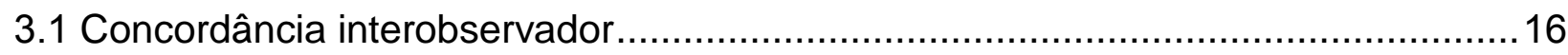

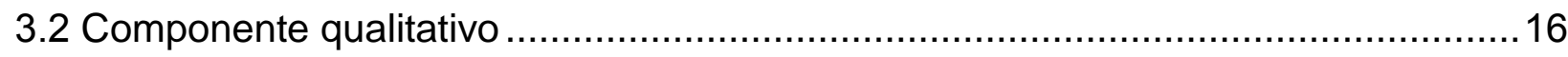

3.3 Componente quantitativo: diâmetros anteroposterior e laterolateral ....................... 18

3.4 Componente quantitativo: relação sinal / ruído estimada ..................................... 19

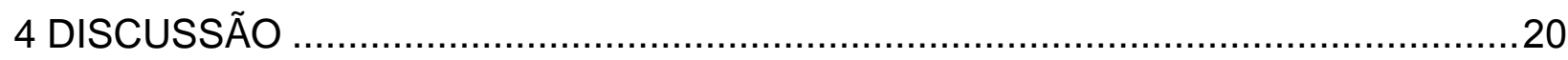

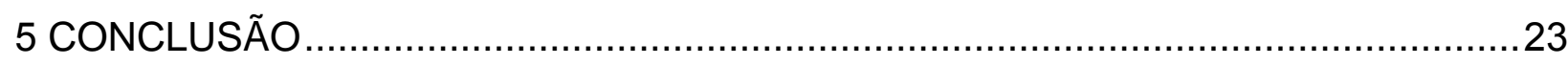

6 ANEXO

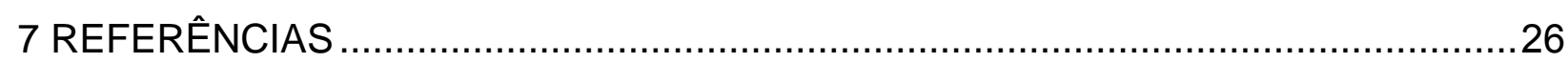

Abstract

Apêndice 


\section{Lista de abreviaturas}

$\begin{array}{ll}\text { ADC } & \text { Apparent diffusion coefficient } \\ \text { AP } & \text { Anteroposterior } \\ \text { Art } & \text { Artefatos } \\ \text { CaP } & \text { Câncer de próstata } \\ \text { DCP } & \text { Definição dos contornos prostáticos } \\ \text { DI } & \text { Distorção da imagem } \\ \text { DWI } & \text { Diffusion weighted imaging } \\ \text { FOCUS } & \text { Field of view optimized and constrained undistorted single shot } \\ \text { FOV } & \text { Field of view } \\ \text { IVIM } & \text { Intravoxel incoeherent motion } \\ \text { LL } & \text { Laterolateral } \\ \text { PIRADS v.2 } & \text { Prostate imaging reporting and data system version 2 } \\ \text { PSA } & \text { Antígeno prostático específico (Prostatic Specific Antigen) } \\ \text { QG } & \text { Qualidade global } \\ \text { RC/DAZ } & \text { Resolução de contraste/definição da anatomia zonal } \\ \text { RLF/GCLF/DLLF } & \text { Relação lesão fundo/grau de confiança na detecção de lesões } \\ & \text { focais/definição dos limites de lesões focais } \\ \text { RM } & \text { Ressonância magnética } \\ \text { RMp } & \text { Ressonância magnética da próstata } \\ \text { RMbp } & \text { Ressonância magnética biparamétrica da próstata } \\ \text { RMmp } & \text { Ressonância magnética multiparamétrica da próstata } \\ \text { RSR } & \text { Relação sinal/ruído } \\ \text { RSRe } & \text { Relação sinal/ruído estimada }\end{array}$




\section{Resumo}

Objetivo: Comparar qualitativamente e quantitativamente uma nova sequência de difusão biexponencial IVIM com a sequência padrão monoexponencial FOCUS em exames de ressonância magnética da próstata. Método: Estudo com coleta prospectiva e análise transversal aprovado pelo comitê de ética em pesquisa institucional. Entre agosto de 2017 e novembro de 2017 foram recrutados 70 pacientes que realizaram ressonância magnética multiparamétrica da próstata por suspeita de neoplasia prostática, incluindo sequências de difusão monoexponencial FOCUS e biexponencial IVIM. As imagens obtidas por ambas as sequências foram julgadas por 2 leitores independentes quanto a critérios de avaliação subjetiva/qualitativa (qualidade global, relação sinal/ruído, resolução de contraste/definição da anatomia zonal, relação lesão fundo/grau de confiança na deteç̧ão de lesões focais/definição dos limites de lesões focais, definição dos contornos prostáticos, distorção da imagem e artefatos) e objetiva/quantitativa (diâmetros anteroposteriores e laterolaterais das próstatas, e relação sinal/ruído estimada), com comparação dos resultados por análise estatística (concordância interobservador por meio do coeficiente de Gwet, análise das variáveis qualitativas pelo teste de Stuart-Maxwell para homogeneidade marginal e análise das variáveis quantitativas através de testes de Wilcoxon para amostras pareadas). Resultados: Dos pacientes inicialmente selecionados, 4 foram excluídos, sendo a casuística final de 66 pacientes. Foi verificada boa/excelente concordância entre os observadores para os critérios de análise subjetiva (exceto para um critério). Na análise qualitativa a classificação de qualidade boa ou excelente foi maior para o FOCUS, à exceção de uma única categoria, com evidências de diferenças estatisticamente significantes para qualidade global DWI, relação sinal-ruído DWI e relação sinal-ruído ADC. $\mathrm{Na}$ análise dos dados quantitativos a sequência FOCUS apresentou menor variabilidade dos diâmetros anteroposteriores, traduzindo menor distorção das imagens, e melhor relação sinal/ruído estimada. Conclusão: A sequência padrão monoexponencial FOCUS mostrou-se superior qualitativa e quantitativamente à sequência IVIM. 


\section{INTRODUÇÃO}

\subsection{Anatomia prostática}

A próstata faz parte do sistema reprodutivo masculino e situa-se na porção inferior da pelve, em contato com a bexiga superiormente, com reto posteriormente e com o diafragma urogenital inferiormente. Tipicamente em um adulto jovem seu peso varia entre 20 e $40 \mathrm{~g}$, com um tamanho médio de $3 \times 4 \times 2 \mathrm{~cm}$, sendo a maior glândula acessória deste sistema. Ela é composta por tecido glandular $(70 \%)$ e tecido fibromuscular ou estromal $(30 \%)$ e é responsável pela produção de aproximadamente $30 \%$ do volume de fluido seminal. ${ }^{(1)}$ É basicamente dividida em três zonas distintas com diferentes origens embriológicas: zona periférica, zona central e zona de transição. (2)

A zona periférica perfaz aproximadamente $70 \%$ do volume prostático total em um adulto jovem, envolvendo a uretra distal no ápice prostático e estendendose posterolateralmente à base. A zona periférica está ausente na porção mais anterior da próstata, área que é ocupada pelo estroma fibromuscular anterior. A zona central perfaz cerca de $25 \%$ do volume prostático e contém os ductos ejaculatórios. Por fim, a zona de transição ocupa os $5 \%$ remanescentes, predominantemente anterolateralmente à uretra proximal ${ }^{(3)}$ (Figura 1).

No adulto, e principalmente no homem idoso, ocorre uma inversão desta relação, secundária ao grande aumento volumétrico da zona de transição devido à presença de nódulos de hiperplasia benigna, frequentemente passando a zona de transição a ocupar a maior parte do volume prostático, rechaçando e determinando afilamento da zona periférica. Devido esta particularidade do progressivo aumento da zona de transição com a idade, assumindo grandes dimensões, se torna difícil a distinção da mesma com a zona central, sendo o conjunto de ambas denominado genericamente de "glândula central" ou "glândula interna". ${ }^{(4)}$ 


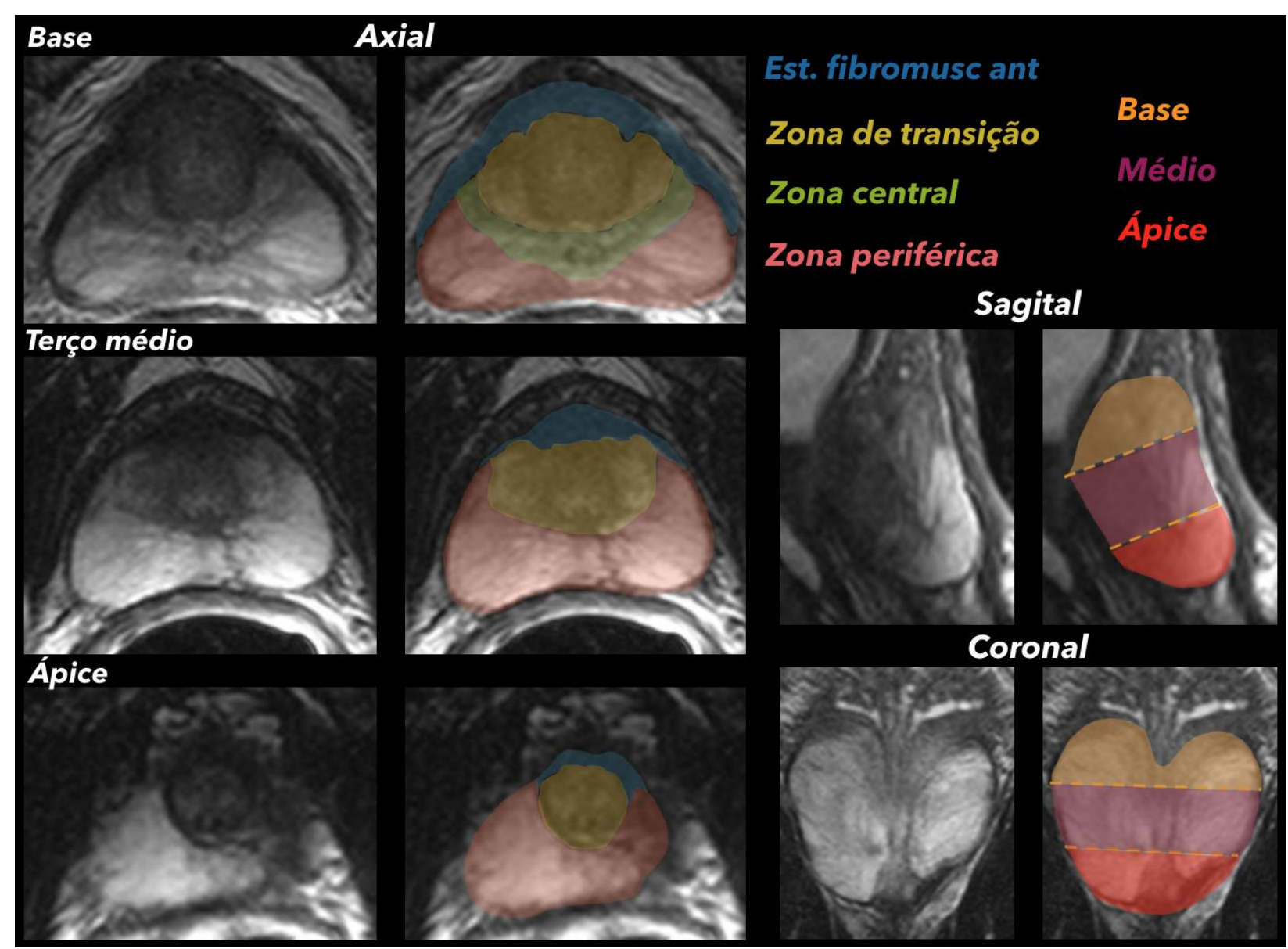

Est. fibromusc ant: estroma fibromuscular anterior.

Figura 1. Anatomia prostática

\subsection{Câncer de próstata}

O câncer de próstata ( $\mathrm{CaP})$ é o mais frequente em homens, excetuando-se os tumores de pele não melanoma, e a segunda causa de morte nessa população. Estima-se que em 2018 no Brasil serão diagnosticados 68.220 novos casos, com cerca de 14.000 mortes anuais relacionadas à doença. ${ }^{(5)}$

Tem bom prognóstico quando diagnosticado precocemente, ou seja, confinado à próstata (estadios 1 e 2) ou com acometimento apenas regional (estadio 3) apresentando taxas sobrevida de $94 \%$ no primeiro ano, $85 \%$ em 5 anos e $84 \%$ em 10 anos, sendo que a taxa de sobrevida câncer específica pode chegar a 100\% em 5 anos e 95\% em 15 anos. Contudo, caso detectado tardiamente, ou seja, quando já estão presentes metástases à distância, a taxa de sobrevida em 5 anos cai para 28-30\%, fato que torna incontestável a importância do diagnóstico precoce desta doença. ${ }^{(6,7)}$ 
O adenocarcinoma é o subtipo histológico mais frequente, responsável por $95 \%$ dos $\mathrm{CaP}{ }^{\left({ }^{(8)}\right.}$ Eles se desenvolvem nos ácinos dos ductos prostáticos, com maior frequência na zona periférica/porção posterior da glândula (70\%), e menos comumente na "glândula central" $(30 \%) .{ }^{(9)}$

\subsection{Manejo atual do câncer prostático}

$\mathrm{O}$ adequado manejo do $\mathrm{CaP}$ envolve detecção, localização e estadiamento da lesão, para que se possa planejar da maneira mais completa possível a melhor estratégia terapêutica a ser adotada. ${ }^{(10)}$ A detecção precoce e localização precisa da lesão são fatores fundamentais no desfecho final obtido, ou seja, quanto mais precocemente detectado, menor será o tamanho do tumor e maiores serão as possibilidades terapêuticas, incluindo estratégias que possam inclusive levar à cura. Além disso, quanto mais precisa a localização, mais efetivo será o tratamento, com menores complicações, menor morbidade e, consequente, maior qualidade de vida após o tratamento.

É exatamente neste ponto em que a ressonância magnética da próstata ( $\mathrm{RMp}$ ) passa ter papel cada vez mais importante. Atualmente, ainda, as diretrizes das maiores sociedades de urologia do mundo preconizam como estratégia no manejo do CaP rastreamento individualizado com antígeno prostático específico (PSA - Prostatic Specific Antigen) e toque retal, e caso ao menos um destes dois estejam alterados prossegue-se com a biópsia por ultrassonografia, randômica, por amostragem aleatória da próstata. ${ }^{(11,12)}$ Ou seja, a partir do momento em que suspeitamos da presença de um tumor na próstata, em geral biopsia-se este órgão às cegas, sem saber efetivamente onde está a lesão e se a mesma foi amostrada adequadamente nos fragmentos obtidos. No manejo de outras neoplasias primeiro localizamos a lesão suspeita, para então depois fazermos uma biópsia dirigida à mesma, e desta forma amostrá-la adequadamente. Outro grande problema da biópsia aleatória é que, além de muitas vezes não amostrarmos a lesão principal ou lesão índice, acabamos encontrando outras lesões consideradas clinicamente "não significantes". Com o envelhecimento, todo tecido prostático sofre displasias/transformações "pré-neoplásicas", mas a imensa maioria dessas alterações são indolentes, ou seja, não terão impacto clínico pois o paciente morrerá antes por 
outros motivos. O fato é que, ao detectarmos estas lesões oneramos o processo, seja com aumento na ansiedade do paciente por saber que as têm, ou seja por intervenções desnecessárias em lesões que não teriam implicação na qualidade de vida ou longevidade do paciente. ${ }^{(10)}$

Estratégias mais modernas e precisas se utilizam da RMp para mapear áreas suspeitas para CaP, consequentemente obtendo resultados de biópsia com maior positividade e mais fidedignos, ou seja, capazes de amostrar adequadamente as lesões de maior agressividade/lesões índice e diminuir a taxa de "sobrediagnóstico" de lesões sem importância clínica, permitindo um planejamento mais adequado e abrangente da estratégia terapêutica a ser adotada. ${ }^{(13)}$

Portanto, atualmente a RMp, somada à avaliação clínica e dosagem dos níveis séricos de PSA, tem papel fundamental no processo diagnóstico, permitindo melhor detecção, estadiamento e planejamento terapêutico. ${ }^{(8)}$

\subsection{Ressonância magnética da próstata}

Tradicionalmente, a RMp baseava-se somente em sequências morfológicas, sobretudo sequências ponderadas em T2 (Figura 2a), implicando em limitação diagnóstica. Avanços nos métodos de imagem acrescentaram a essa avaliação básica sequências funcionais, incluindo a difusão (Figura 2c), o mapa de coeficiente de difusão aparente (Figura 2d) e as sequências dinâmicas pós-contraste (Figura 2b), com excelentes resultados para avaliação da próstata, promovendo maior acurácia na detecção e estadiamento de tumores. ${ }^{(8,14)}$ Atualmente, essas sequências fazem parte do protocolo habitual, daí o nome, mais utilizado nos dias de hoje, de ressonância magnética multiparamétrica da próstata $(\mathrm{RMmp}) .{ }^{(15)}$ No nosso serviço, a RMmp se incorporou ao algoritmo de rastreamento de CaP em casos selecionados desde 2013. Atualmente é realizada conforme indicação clínica, preferencialmente em aparelho de alto campo magnético (3 Tesla), sem bobina endorretal. 


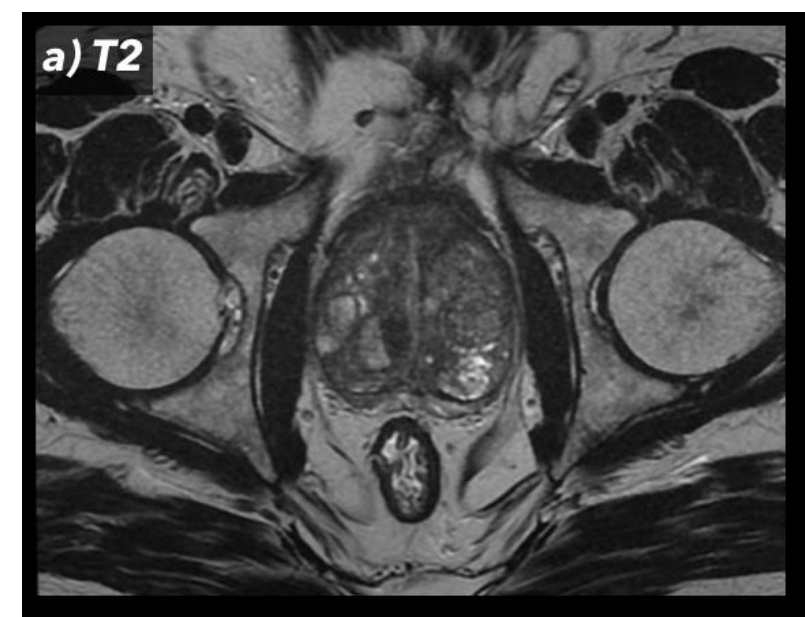

\section{b) Dinâmica pós-contraste}

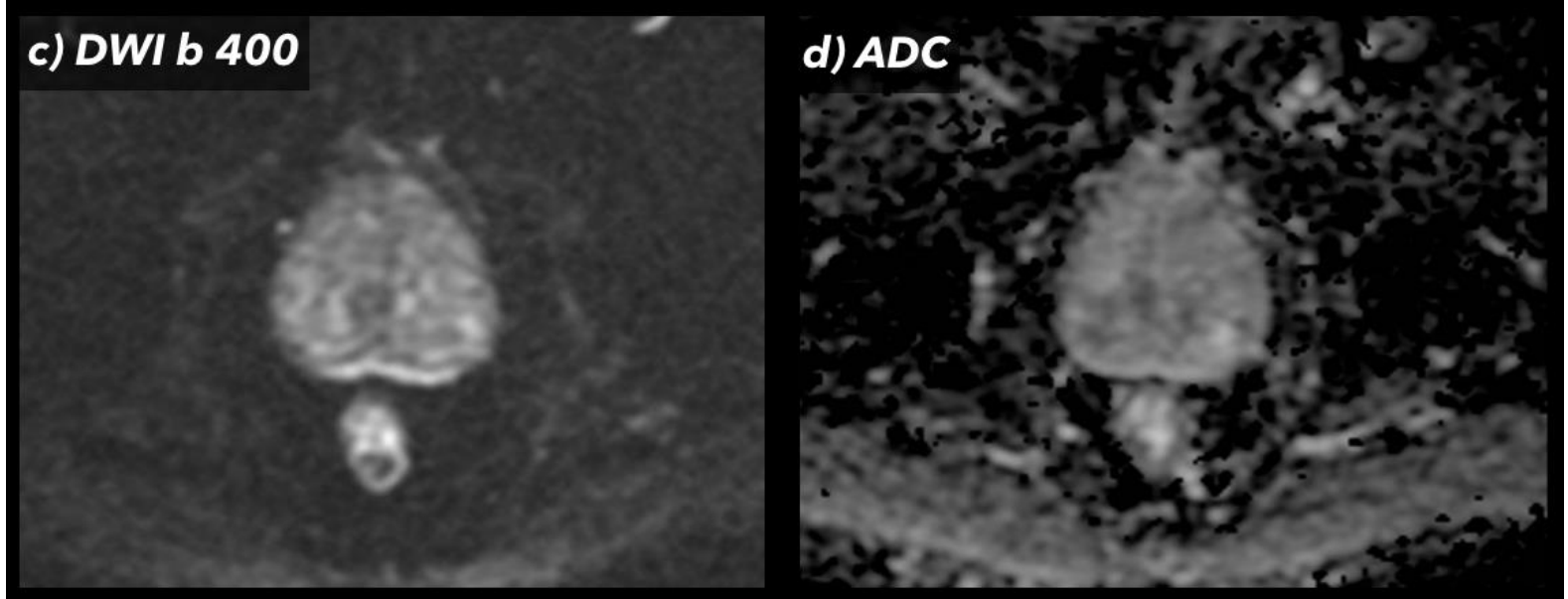

a) aquisição axial T2, b) aquisição dinâmica pós-contraste, c) aquisição em difusão com valor de b de 400, d) mapa de coeficiente de difusão aparente ADC.

Figura 2. Protocolo RMmp

Ao falarmos de diagnóstico do CaP através da RM é importante citar o sistema estruturado e padronizado de relatórios de ressonância magnética de próstata, o PIRADS v.2 (Prostate Imaging Reporting and Data System, version 2). ${ }^{(16)}$ Trata-se de um esquema de relatórios estruturados concebido para a avaliação préterapêutica dos pacientes sob suspeita clínica que categoriza os achados em uma escala de probabilidade de neoplasia clinicamente significante (Tabela 1). Para que tal relatório possa ser utilizado é necessário a aquisição de sequências ponderadas em T2, em difusão e dinâmicas pós-contraste. 
Tabela 1. Interpretação PIRADS v2

\begin{tabular}{|c|c|c|c|}
\hline $\begin{array}{l}\text { Escore } \\
\text { PIRADS }^{\circledR} \text { v2 }\end{array}$ & Descrição & $\begin{array}{l}\text { Risco de CaP } \\
\text { clinicamente } \\
\text { significante }\end{array}$ & Conduta \\
\hline 1 & $\begin{array}{l}\text { Muito baixa probabilidade de } \\
\text { CaP clinicamente significante }\end{array}$ & $\sim 3 \%$ & $\begin{array}{l}\text { Mantém conduta/seguimento } \\
\text { clínico }\end{array}$ \\
\hline 2 & $\begin{array}{l}\text { Baixa probabilidade de CaP } \\
\text { clinicamente significante }\end{array}$ & $\sim 10 \%$ & $\begin{array}{l}\text { Mantém conduta/seguimento } \\
\text { clínico }\end{array}$ \\
\hline 3 & $\begin{array}{l}\text { Achados indeterminados para } \\
\text { CaP clinicamente significante }\end{array}$ & $\sim 7-40 \%$ & $\begin{array}{l}\text { Ponderar biópsia dirigida } \mathrm{x} \\
\text { seguimento }\end{array}$ \\
\hline 4 & $\begin{array}{l}\text { Moderada probabilidade de CaP } \\
\text { clinicamente significante }\end{array}$ & $\sim 65 \%$ & Biópsia dirigida \\
\hline 5 & $\begin{array}{l}\text { Alta probabilidade de } \mathrm{CaP} \\
\text { clinicamente significante }\end{array}$ & $\sim 85 \%$ & Biópsia dirigida \\
\hline
\end{tabular}

CaP: câncer prostático.

Fonte: Traduzido e adaptado de Weinreb JC, Barentsz JO, Choyke PL, Cornud F, Haider MA, Macura KJ, et al. PI-RADS Prostate Imaging - Reporting and Data System: 2015, Version 2. Eur Urol. 2016;69(1):16-40. ${ }^{(16)}$

Além das já mencionadas sequências anatômicas ponderadas em T2, dentro do protocolo de RMmp temos as sequências dinâmicas pós-contraste, ou seja, adquiridas após injeção intravenosa de contraste paramagnético, que fazem parte e são necessárias para o uso do PIRADS v2. Estas sequências baseiam-se no princípio de que tumores de modo geral apresentam neovascularização/aumento nos capilares teciduais (ou seja, são hipervascularizados) e tal característica é evidenciada pelo reforço de sinal nas áreas tumorais após injeção endovenosa do contraste. Porém, atualmente o uso do contraste na RMp é bastante controverso, com alguns autores advogando que o mesmo acrescenta muito pouco à capacidade do método em detectar e estimar o grau de probabilidade de neoplasias clinicamente significantes ${ }^{(17)}$ e outros defendendo sua manutenção por agregar bastante ao método. ${ }^{(18)}$ Outro aspecto importante a ser considerado é que o uso de contraste paramagnético onera o processo, seja por acrescentar tempo de aquisição ao exame e pelo próprio custo do fármaco em si, ou pelo risco de eventos adversos relacionados ao uso do mesmo, como reações alérgicas e deposição cerebral. ${ }^{(19)}$ Desta forma, caso não fosse mais necessário o seu uso, por exemplo pelo desenvolvimento de uma nova sequência, o acesso à RMmp poderia ser ampliado. A perspectiva de realização de RMp sem sequências dinâmicas pós-contraste deu origem a um novo termo: ressonância magnética biparamétrica da próstata (RMbp), fazendo referência às sequências ponderadas em T2 e difusão. Tal protocolo tem como principais vantagens ser mais rápido, mais barato e inócuo, com um enorme potencial de uso para rastreamento populacional. $^{(20-26)}$ 


\subsection{Difusão na ressonância magnética da próstata}

Com o avanço e consolidação da RMmp as sequências ponderadas em difusão (DWI - Diffusion Weighted Imaging), bem como seus mapas de coeficiente de difusão aparente (ADC - Apparent Diffusion Coefficient), assumiram incontestavelmente papel cada vez mais importante no protocolo de exame. Elas se baseiam no princípio da difusão das moléculas de água, sendo capazes de mensurar essa movimentação. As moléculas de água, fora do organismo, movimentam-se livremente, de forma aleatória, o que não ocorre nos diferentes tecidos corporais, visto a movimentação ser afetada pela interação da água com as membranas celulares e macromoléculas, podendo ocorrer nos espaços intracelular, extracelular e intravascular. Condições que promovem a redução da movimentação dessas moléculas, como alta celularidade, condicionam maior restrição à difusão, o que pode ser avaliado pela difusão por meio da alteração da intensidade de sinal. Isso é o que ocorre nos tumores, dentre eles o CaP. Na RM, é possível ajustarmos a sensibilidade das sequências de difusão alterando um de seus parâmetros, denominado valor de $b$, que é modificado principalmente, mas não exclusivamente, pela alteração na amplitude do gradiente. ${ }^{(27-30)}$ Basicamente, com a utilização de alguns valores de $b$ diferentes na avaliação dos tecidos, tem-se a difusão com análise monoexponencial, rotineiramente já utilizada nos protocolos de RM de próstata, inclusive em nosso serviço. A partir das aquisições fonte de difusão ponderadas em diferentes valores de $b$, ou seja, adquiridas variando-se a amplitude do gradiente, os mapas de $A D C$ são extrapolados automaticamente pelos softwares integrados aos aparelhos, e destes é possível obter dados quantitativos da impedância ou difusividade tecidual.

É teorizado que o componente rápido da difusão em baixos valores de $b$ mostra o movimento das moléculas no fluxo capilar (componente de perfusão), refletindo o grau de angiogênese e em última análise de perfusão naquele tecido, e que o componente lento da difusão em valores de $b$ relativamente altos é relacionado com a celularidade do tecido (componente de difusão). ${ }^{(31)}$ No CaP, existe um aumento da celularidade do tecido com consequente redução no movimento livre das moléculas de água e também um aumento na perfusão do sangue secundário a neo-angiogênese, que leva a aumento dos capilares teciduais. Dessa forma, a difusão fornece importante parâmetro físico utilizado para diferenciar tecido prostático maligno de benigno, 
podendo inclusive predizer o grau de agressividade daquele tumor, uma vez que quanto maior o desarranjo celular, maior a restrição à difusão. No entanto, em algumas doenças benignas também existe o aumento da perfusão sanguínea devido a alterações inflamatórias, o que pode causar uma restrição à difusão apesar de não haver mudança na celularidade tecidual, gerando um resultado falso positivo da difusão convencional, monoexponencial. ${ }^{(29,32-36)}$

Em 1988, Le Bihan et al. ${ }^{(31)}$ descreveram a técnica de difusão biexponencial, também denominada IVIM (Intravoxel Incoherent Motion), que em teoria é capaz de separar os componentes de perfusão e de difusão, podendo então aferir de maneira mais precisa isoladamente o componente de "verdadeira restrição à difusão", além de avaliar o componente de perfusão, avaliando indiretamente a vascularização capilar daquele tecido ou lesão.

A difusão biexponencial consiste na realização de múltiplos valores de $b$ com avaliação biexponencial para obtenção de alguns parâmetros e pode ser representado pela seguinte equação biexponencial:

$$
S(b)=S_{0}\left[f e^{-b D^{*}}+(1-f) e^{-b D f}\right]
$$

Onde:

- S(b): é a intensidade de sinal para um determinadao valor de $b$

- $f$. é a fração de perfusão

- $D^{*}$ ou $D_{\text {slow: }}$ reflete à difusidade tecidual, ou seja, o componente de "restrição verdadeira";

- Df ou $D_{\text {fast: }}$ reflete a perfusão microcapilar; ou seja, o componente de "pseudodifusão".

O desenvolvimento e pesquisa da difusão biexponencial surgiu e ainda é maior na área da neurorradiologia, com trabalhos avaliando desde a perfusão cerebral global em determinadas situações, a estudos mais dirigidos à análise de parâmetros tumorais. ${ }^{(37-41)}$

$\mathrm{Na}$ radiologia abdominal alguns estudos mostraram bons resultados da difusão biexponencial em diferentes cenários clínicos. ${ }^{(42,43)}$

Em relação à aplicação do IVIM nos exames de RMp as publicações na literatura vigente também são todas experimentais, com resultados discordantes 
entre si ou não reprodutíveis. ${ }^{(31,44-47)}$ Os protocolos de aquisição variam nas diferentes publicações, de modo que até o momento não existe nenhum protocolo bem estabelecido e aceito, replicável em outras instituições e com maior potencial de uso clínico.

Devemos citar também que desde as primeiras publicações a respeito da técnica no final da década de 1980 por Le Bihan et al. ${ }^{(31,37)}$ a teórica capacidade de realmente reproduzir e aferir o componente de perfusão tecidual pelo IVIM é controversa, questionada por alguns autores ${ }^{(48,49)}$ e defendida por outros. ${ }^{(31,50,51)}$ Porém, à luz do conhecimento atual e considerando-se todas as publicações existentes, é difícil questionar o embasamento teórico da técnica, já bem estabelecido por vários estudos pré-clínicos em animais. ${ }^{(52-58)}$

Outros processos fisiológicos que também geram "fluxo tecidual" influenciam o componente rápido da difusão ou fração de perfusão medida pelo IVIM, causando interferências na percepção do fluxo vascular tecidual, como por exemplo, secreção glandular e fluxo ductal. ${ }^{(59,60)}$ Tais processos atuam como fatores de confusão, talvez explicando ao menos em parte alguns dos maus resultados apresentados pelo método.

A maioria dos estudos existentes avaliou quais parâmetros quantitativos seriam ou não reprodutíveis, por meio de análise dos valores obtidos a partir das imagens adquiridas com protocolo de difusão biexponencial, como por exemplo, valores dos mapas de ADC de áreas comprovadamente tumorais. ${ }^{(44,45,51)}$

No nosso trabalho, buscamos primeiramente estabelecer um protocolo de difusão biexponencial factível, para então compararmos a qualidade com a difusão monoexponencial já bem estabelecida e sabidamente de grande valia na prática clínica. Os trabalhos publicados estudando-se a técnica de difusão biexponencial IVIM variam substancialmente quanto aos protocolos utilizados na aquisição das imagens, isto é claramente evidenciado no artigo de revisão publicado por Koh et al. ${ }^{(50)}$ onde são listados ao menos 12 protocolos distintos. Este é um ponto crítico da técnica. Não existe consenso bem estabelecido em relação ao número de valores de $b$, tampouco das magnitudes desses, necessários para que possamos obter medidas clínicas confiáveis e reprodutíveis. ${ }^{(50)} \mathrm{A}$ aquisição das imagens com valores de $b$ baixos é mais susceptivel a distúrbios na relação sinal/ruído (RSR), sendo mais um desafio para o amplo uso da técnica, com alguns trabalhos inclusive demonstrando essa limitação. ${ }^{(61)}$ Tal peculiaridade técnica na aquisição das imagens suscitou 
trabalhos mais recentes que avaliaram diferentes modelos de ajuste para difusão biexponencial, em outras áreas ${ }^{(62)}$ e especificamente na imagem do $\mathrm{CaP},{ }^{(63)}$ buscando averiguar a executabilidade e reprodutibilidade dos protocolos.

Portanto, o intuito do presente estudo é testar um protocolo de difusão biexponencial em exames de próstata comparando-o ao protocolo de difusão monoexponencial padrão. Uma vez bem estabelecido, factível e reprodutível, com qualidade de imagem ao menos comparável ao protocolo padrão atualmente em voga, o modelo biexponencial de difusão teria, em tese a capacidade de agregar a informação da perfusão tecidual por avaliar separadamente os componentes de perfusão e de difusão, o que talvez promova um aumento na detecção com uma melhor caracterização do $\mathrm{CaP}$, aumentando em última análise a acurácia do método. Provando-se esta hipótese, poderíamos advogar sobre a possibilidade de não mais usarmos rotineiramente o contraste endovenoso em todos os exames de RMp, uma vez que a nova sequência já trará informações a respeito da vascularização das lesões.

No ponto final de todos esses avanços, busca-se um aumento da acurácia na detecção e caracterização de lesões suspeitas para câncer clinicamente significante na próstata, permitindo indicar com maior segurança e precisão o local exato de maior suspeição a fim de serem realizadas biópsias dirigidas para tais áreas suspeitas, aumentando a efetividade da intervenção médica em todo o processo, evitando intervenções desnecessárias, com consequente redução do desconforto e ansiedade do paciente e do custo global no diagnóstico e manejo do CaP.

\subsection{Objetivo}

1. Testar um protocolo de difusão biexponencial (IVIM), comparando-o ao protocolo padrão de difusão monoexponencial (FOCUS) rotineiramente utilizado. 


\section{MÉTODOS}

\subsection{População}

Neste estudo com coleta prospectiva e análise transversal foram recrutados entre agosto de 2017 e novembro de 201770 pacientes consecutivamente submetidos ao exame de RMp sem bobina endorretal, única e exclusivamente por indicações clínicas, ou seja, suspeita clínica de CaP clinicamente significante, em pacientes sem histórico de CaP prévio, secundário a aumento nos níveis de PSA e/ou toque retal alterado, sendo este o único critério de inclusão, sem a interferência dos participantes do estudo e sem outros critérios de seleção previamente estabelecidos. Os critérios de exclusão foram contraindicações ao método (uso de dispositivos não compatíveis com a RM, claustrofobia, entre outros), problemas técnicos na aquisição ou no pós-processamento das imagens e análise por apenas um dos leitores. Salientase que o presente estudo não alterou a indicação da RMp.

\subsection{Protocolo da ressonância magnética}

Foi implementado no aparelho GE Discovery MR 750w 3.0 Tesla a sequência em teste (difusão IVIM ou difusão biexponencial) (Anexo 1), e a mesma adquirida adicionalmente a todas as demais sequências já estabelecidas e usadas rotineiramente no protocolo da instituição (ou seja, foram adquiridos em todos os exames 2 sequências distintas de difusão, a sequência monoexponencial já bem estabelecida e tida como referência, aqui denominada difusão FOCUS e a sequência de difusão biexponencial teste, aqui denominada difusão IVIM (Figura 3). As aquisições foram feitas com o uso de bobina de superfície (bobina cardíaca), sem bobina endorretal.

Os parâmetros da difusão IVIM foram otimizados por meio de extensa revisão da literatura, testes em voluntários sadios e intercâbio de informações com a equipe de desenvolvimento da sequência, para assegurar uma boa RSR mesmo nos valores de $b$ mais altos. $O$ tempo de aquisição total da sequência teste IVIM foi de 8 minutos e 12 segundos. 


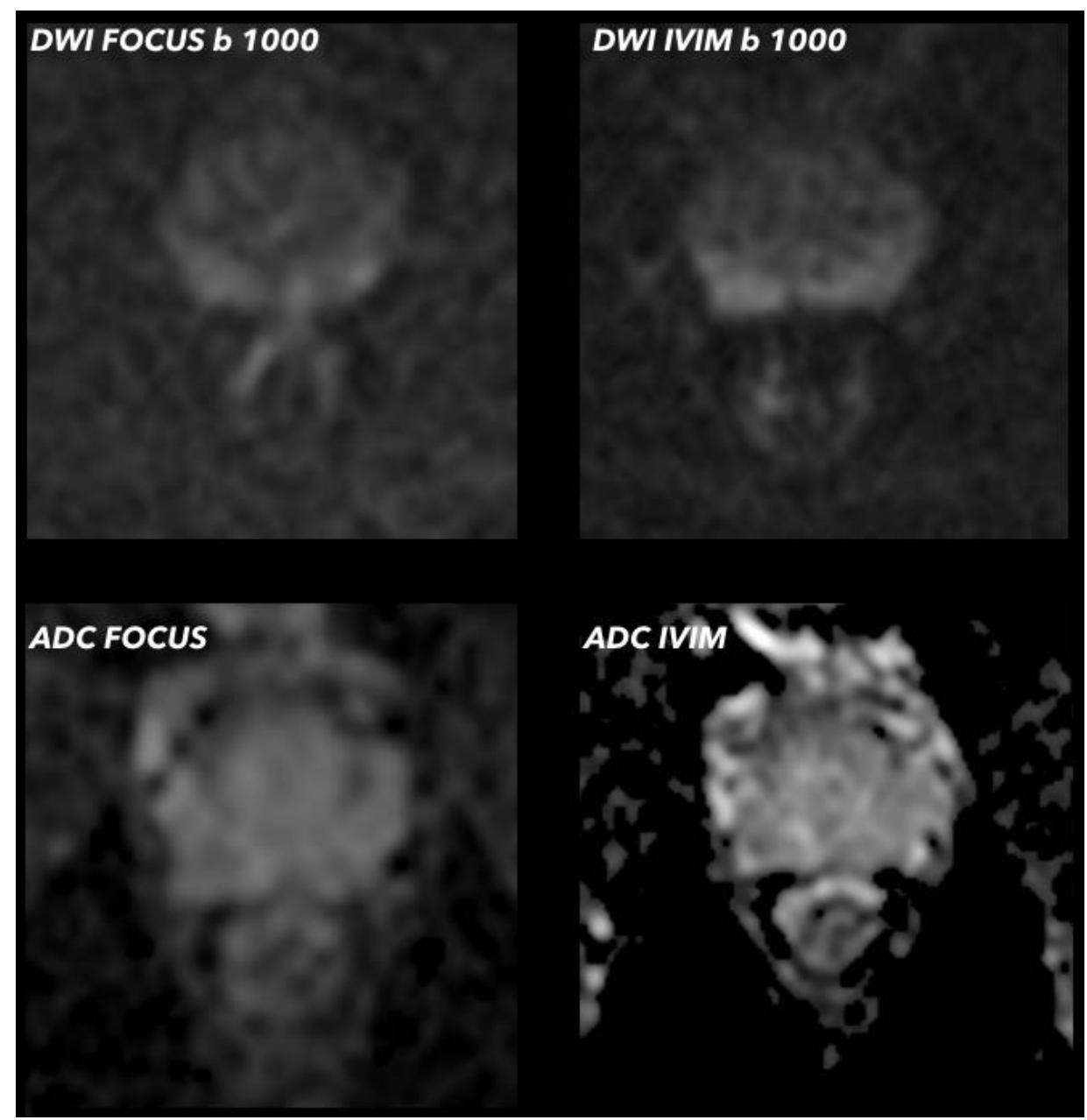

Figura 3. Difusão e ADC monoexponencial padrão FOCUS $x$ difusão e ADC biexponencial teste IVIM

\subsection{Métodos de análise}

A comparação da qualidade das duas sequências foi subdividida em 2 componentes, um componente qualitativo/subjetivo e um componente quantitativo/objetivo por meio de medidas realizadas nas imagens.

\subsubsection{Componente qualitativo}

As imagens foram analisadas por dois radiologistas com mais de 5 anos experiência em RMp, sempre comparando-se os valores de $b$ mais altos de cada sequência e os respectivos mapas de $A D C$, com base nos seguintes critérios: qualidade global (QG), RSR, resolução de contraste/definição da anatomia zonal (RC/DAZ), relação lesão fundo/grau de confiança na detecção de lesões 
focais/definição dos limites de lesões focais (RLF/GCLF/DLLF), definição dos contornos prostáticos (DCP), distorção da imagem (DI) e artefatos (Art).

Tais critérios foram julgados de acordo com a seguinte escala 1: muito ruim, 2: ruim, 3: moderado, 4: bom e 5: excelente. ${ }^{(64)}$

Antes do início da leitura houve reuniões e sessões de treinamento para que ambos os radiologistas estivessem alinhados em relação ao conceito de cada parâmetro avaliado.

\subsubsection{Componente quantitativo}

Foram realizadas medidas para avaliar quantitativamente a presença e o grau de distorção das imagens e avaliar de maneira estimada a RSR.

Para mensuração do grau de distorção das imagens foram realizadas medidas dos maiores diâmetros anteroposteriores (AP) e laterolaterais (LL) da próstata nas sequências axial T2 (Figura 4), difusão FOCUS e difusão IVIM. Sabidamente as imagens obtidas por sequências ponderadas em T2 são mais anatômicas e menos susceptíveis a distorções, e portanto as medidas nesta sequência foram consideradas padrão e as variações nas sequências de difusão em relação a sequência T2 foram avaliadas de modo a saber em qual das duas sequências de difusão (padrão x teste) houve maior distorção.

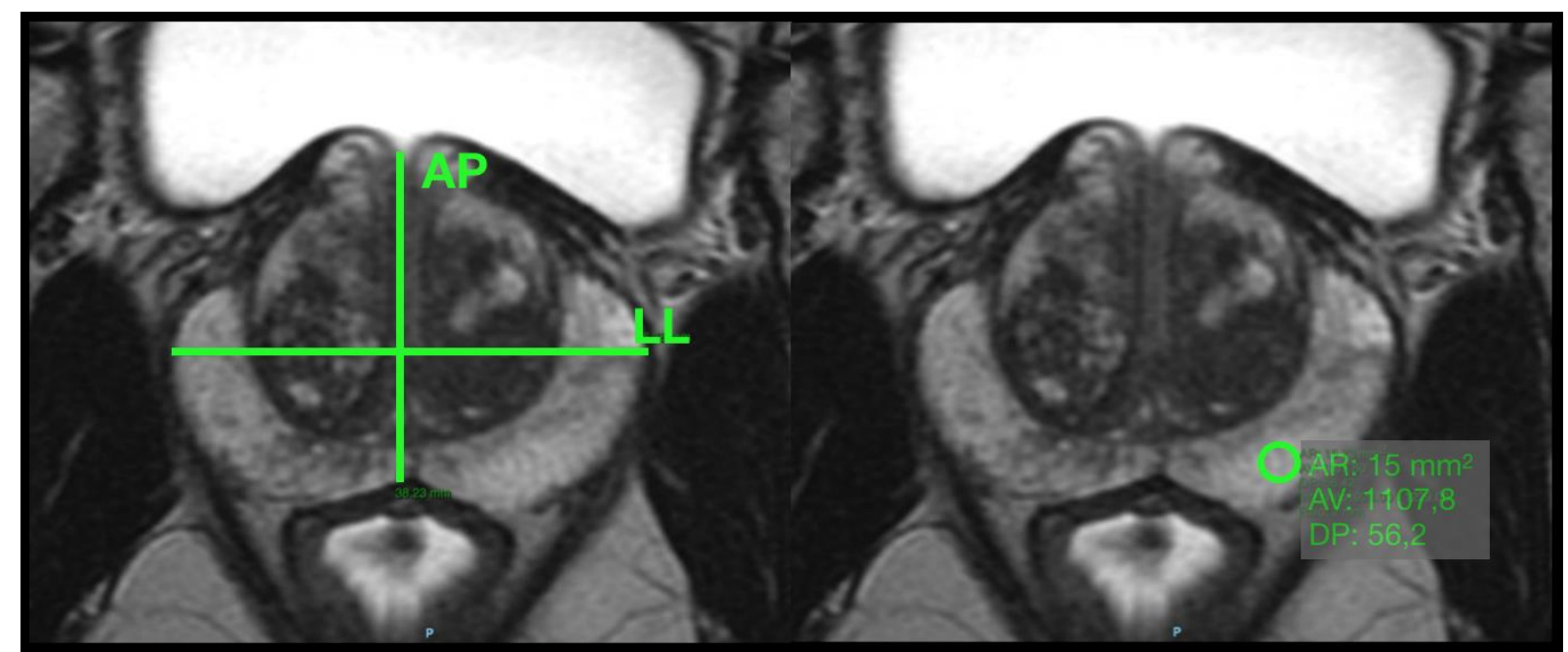

Figura 4. Diâmetros AP e LL e exemplo de posicionamento do ROI para obtenção da RSRe 
A RSR de imagens de RM tradicionalmente é obtida comparando-se o sinal de um determinado tecido em relação ao sinal do ar (em teoria nulo). ${ }^{(65)}$ No entanto, isso não é possível na maioria dos exames de próstata, incluindo ambas as nossas sequências de difusão (FOCUS e IVIM), pois o campo de visão (FOV - Field of View) pequeno não inclui ar, não sendo possível tal relação. A alternativa adotada foi utilizar a relação sinal/ruído estimada (RSRe), que consiste em uma relação entre a média e o desvio padrão da intensidade de sinal dos voxels de cada sequência. ${ }^{(66,67)}$ Para tanto foram posicionados ROl's nas zonas periféricas (com área padrão de $15,3 \mathrm{~mm}^{2}$, média de $14,3 \mathrm{~mm}^{2}$, variando de 5,5 a $15,3 \mathrm{~mm}^{2}$ ) respeitando a seguinte metodologia: posicionados a partir das imagens axiais T2 em regiões relativamente pouco alteradas (Figura 4), sempre evitando lesões focais e replicados de maneira idêntica (mesma área e localização) para as sequências de difusão padrão e teste IVIM, e seus respectivos mapas ADC. Tal medida de área dos ROI's foi arbitrariamente estabelecida de modo que todos os critérios de posicionamento citados acima fossem cumpridos. A partir dos valores obtidos dos mapas ADC foi calculada a RSRe.

\subsection{Análise estatística}

Seguindo a metodologia empregada a análise estatística também foi dividida em dois grupos de modo a avaliar separadamente os componentes qualitativo e quantitativo.

\subsubsection{Componente qualitativo}

Como primeiro passo buscamos avaliar a concordância entre os observadores. Para tanto os dados foram dispostos em tabelas de dupla entrada por frequências absolutas e relativas à amostra total. Para avaliação da concordância entre os leitores foi utilizado o coeficiente de concordância de Gwet, ${ }^{(68)}$ apropriado nos casos em que a proporção de respostas é concentrada em alguma das categorias. Para a interpretação do coeficiente de concordância entre avaliadores utilizamos a seguinte classificação proposta por Altman: ${ }^{(69)}$ ruim (menor ou igual a 0,20), razoável (entre 0,21 e 0,40), moderada (entre 0,41 e 0,60), boa (entre 0,61 e 0,80) e excelente (acima de 
0,81). Uma vez estabelecida concordância satisfatória entre os dois leitores, foi sorteado um deles por meio de programa estatístico apropriado ("R: The R Project for Statistical Computing") para então realizarmos a comparação entre as duas sequências (FOCUS x IVIM). Essa comparação foi realizada pelo teste de Stuart-Maxwell para homogeneidade marginal (ou teste de McNemar generalizado), ${ }^{(70)}$ por ser apropriado para comparações pareadas de variáveis qualitativas com mais de duas categorias.

\subsubsection{Componente quantitativo}

As comparações entre as sequências IVIM e FOCUS quanto às variáveis quantitativas foram realizadas por meio de testes de Wilcoxon para amostras pareadas, considerando para as análises nível de significância de 5\%.

\subsection{Aspectos éticos}

Nosso estudo foi aprovado pelo comitê de ética em pesquisa desta instituição, e previamente a cada exame adquirido foi obtido consentimento livre e esclarecido dos pacientes. Possui desenho prospectivo, porém isto não interferiu na indicação do exame de RM e não acarretou nenhum risco potencial ao paciente. $\mathrm{O}$ único contratempo imposto foi a necessidade de permanência por alguns minutos adicionais no aparelho de RM, necessário para aquisição da sequência teste adicional. Tal particularidade foi devidamente explicada tanto por escrito no termo de consentimento livre e esclarecido, quanto verbalmente pela equipe assistencial antes da realização do exame para que o mesmo pudesse voluntariamente decidir se gostaria ou não de participar do estudo.

Todas as análises dirigidas a este estudo foram realizadas em um momento distinto da leitura das demais sequências e confecção do relatório radiológico, de modo que a sequência teste não tivesse nenhuma influência sobre 0 mesmo, e a análise fosse focada na comparação de qualidade entre as duas sequências. 


\section{RESULTADOS}

Dos 70 pacientes inicialmente incluídos, 2 foram excluídos por artefatos relacionados à aquisição das imagens (artefatos de susceptibilidade magnética que degradaram igualmente ambas as sequências de difusão) e 2 por critérios técnicos relacionados ao pós-processamento (crash do software ou da estação de trabalho dedicada, não sendo possível gerar os relatórios com os valores para cálculo da RSRe), sendo portanto nossa casuística final composta por 66 pacientes.

\subsection{Concordância interobservador}

A concordância interobservadores na nossa amostra foi boa ou excelente em todas as análises, variando de 0,62 a 0,95, exceto em um único quesito ("RSR ADC" que teve concordância razoável entre os observadores - 0,32) (Apêndice 1).

\subsection{Componente qualitativo}

Para todos os quesitos avaliados, exceto um deles (definição dos contornos prostáticos - DWI), a classificação de qualidade boa ou excelente foi sempre maior para a sequência FOCUS.

No entanto, evidências de diferenças estatisticamente significativas, sempre favorecendo FOCUS em relação ao IVIM, foram observadas em apenas três categorias: qualidade global DWI, RSR DWI e RSR ADC (Tabela 2). 
Tabela 2. Comparações entre IVIM e FOCUS quanto às variáveis qualitativas

\begin{tabular}{l} 
Critério de avaliação qualitativa \\
\hline Qualidade global - DWI - Leitor 1 \\
Ruim/Muito ruim \\
Moderada \\
Bom/Excelente \\
Relação sinal-ruído - DWI - Leitor 1 \\
Ruim/Muito ruim \\
Moderada \\
Bom/Excelente
\end{tabular}

Resolução de contraste / Definição da anatomia zonal - DWI - Leitor 1 Ruim/Muito ruim Moderada Bom/Excelente

Relação Lesão Fundo / Grau de confiança na detecção de lesões focais / Definição dos limites de lesões focais - DWI - Leitor 1

Não se aplica

Ruim/Muito ruim

Moderada

Bom/Excelente

Definição dos contornos prostáticos - DWI - Leitor 1

Ruim/Muito ruim

Moderada

Bom/Excelente

Distorção da imagem - DWI - Leitor 1

Ruim/Muito ruim

Moderada

Bom/Excelente

Artefatos - DWI - Leitor 1

Ruim/Muito ruim

Moderada

Bom/Excelente

Qualidade global - ADC - Leitor 1

Ruim/Muito ruim

Moderada

Bom/Excelente

Relação sinal-ruído - ADC - Leitor 1

Ruim/Muito ruim

Moderada

Bom/Excelente

Resolução de contraste / Definição da anatomia zonal - ADC - Leitor 1

Ruim/Muito ruim

Moderada

Bom/Excelente

Relação Lesão Fundo / Grau de confiança na

detecção de lesões focais / Definição dos limites de lesões focais - ADC - Leitor 1

Não se aplica

Ruim/Muito ruim

\section{IVIM}

$n(\%)$

$0(0,0)$

$8(12,1)$

$58(87,9)$

$0(0)$

15(22,7)

$51(77,3)$

$0(0)$

10(15,2)

$56(84,8)$

$52(78,8)$

$1(1,5)$

$8(12,1)$

$5(7,6)$

$0(0)$

$6(9,1)$

$60(90,9)$

2(3,0)

$9(13,6)$

$55(83,3)$

$1(1,5)$

$10(15,2)$

$55(83,3)$

$2(3,0)$

$17(25,8)$

$47(71,2)$

$7(10,6)$

$33(50,0)$

$26(39,4)$

$2(3,0)$

$19(28,8)$

$45(68,2)$
FOCUS

$n(\%)$

Valor-p

0,046

$0(0)$

$4(6,1)$

62 $(93,9)$

0,013

$0(0)$

$6(9,1)$

$60(90,9)$

0,059

0(0)

$5(7,6)$

$61(92,4)$

$51(77,3)$

$0(0)$

$3(4,5)$

$12(18,2)$

0(0)

$8(12,1)$

$58(87,9)$

0(0)

$10(15,2)$

$56(84,8)$

$1(1,5)$

$9(13,6)$

$56(84,8)$

$0(0)$

$6(9,1)$

$60(90,9)$

$<0,001$

$1(1,5)$

$3(4,5)$

62 $(93,9)$

0,292

$1(1,5)$

$13(19,7)$

$52(78,8)$

0,414

$-$
$52(78,8)$

$1(1,5)$
$50(75,8)$

$0(0)$ 


\begin{tabular}{lccc}
...continuação & & & \\
\hline Moderada & $4(6,1)$ & $4(6,1)$ & \\
$\quad$ Bom/Excelente & $9(13,6)$ & $12(18,2)$ & 0,102 \\
Definição dos contornos prostáticos - ADC - Leitor 1 & & & \\
$\quad$ Ruim/Muito ruim & $1(1,5)$ & $1(1,5)$ & \\
$\quad$ Moderada & $9(13,6)$ & $5(7,6)$ & \\
$\quad$ Bom/Excelente & $56(84,8)$ & $60(90,9)$ & 0,050 \\
Distorção da imagem - ADC - Leitor 1 & & $1(1,5)$ & \\
$\quad$ Ruim/Muito ruim & $2(3,0)$ & $8(12,1)$ & \\
$\quad$ Moderada & $12(18,2)$ & $57(86,4)$ & 0,223 \\
$\quad$ Bom/Excelente & $52(78,8)$ & & \\
Artefatos - ADC - Leitor 1 & & $1(1,5)$ & \\
$\quad$ Ruim/Muito ruim & $2(3,0)$ & $7(10,6)$ & \\
$\quad$ Moderada & $8(12,1)$ & $58(87,9)$ & \\
$\quad$ Bom/Excelente & $56(84,8)$ & $\mathbf{6 6 ( 1 0 0 )}$ & \\
\hline Total & $\mathbf{6 6 ( 1 0 0 )}$ &
\end{tabular}

Para quatro quesitos (relação lesão-fundo DWI, distorção da imagem $D W I$, qualidade global $A D C$ e relação lesão-fundo $A D C$ ), não foi possível realizar teste de hipóteses pois houve uma disparidade/não pareamento das categorias, condição fundamental para análise pelo método empregado no qual as categorias obrigatoriamente necessitam ser pareadas. Isto ocorreu pois apenas a sequência IVIM foi avaliada como ruim/muito ruim, gerando esse despareamento com a sequência FOCUS que sempre foi avaliada como moderada ou bom / excelente nesses quesitos.

\subsection{Componente quantitativo: diâmetros anteroposterior e laterolateral}

Em relação aos diâmetros AP observa-se menor variação na sequência padrão FOCUS em relação à sequência T2 (referência) com os valores variando $-0,8$ a $+0,4$, ou seja menor distorção das imagens no eixo AP na sequência FOCUS quando comparada à sequência IVIM, estatisticamente significante $(p=0,009)$.

Em relação aos diâmetros LL observa-se menor variação na sequência teste IVIM em relação à sequência T2 (referência) com os valores variando 0,2 a $+0,4$, ou seja menor distorção das imagens no eixo LL na sequência IVIM quando comparada à sequência FOCUS, porém sem significância estatística $(p=0,075)$ (Tabela $3)$. 
Tabela 3. Comparação entre IVIM e FOCUS quanto à variação dos diâmetros LL e AP

\begin{tabular}{|c|c|c|c|}
\hline \multirow{2}{*}{ Diâmetro } & \multicolumn{2}{|c|}{ Diferenças em relação à T2 } & \multirow{2}{*}{ Valor-p } \\
\hline & IVIM-T2 & FOCUS-T2 & \\
\hline AP & & & 0,009 \\
\hline Mediana ( $1^{\circ}$ quartil; $3^{\circ}$ quartil) & $0,00(-0,20 ; 0,10)$ & $-0,10(-0,20 ; 0,00)$ & \\
\hline Mínimo; Máximo & $-1,00 ; 0,50$ & $-0,80 ; 0,40$ & \\
\hline LL & & & 0,075 \\
\hline Mediana ( $1^{\circ}$ quartil; $3^{\circ}$ quartil) & $0,10(0,00 ; 0,20)$ & $0,10(0,00 ; 0,20)$ & \\
\hline Mínimo; Máximo & $-0,20 ; 0,40$ & $-0,50 ; 0,50$ & \\
\hline
\end{tabular}

\subsection{Componente quantitativo: relação sinal / ruído estimada}

A comparação entre as sequências FOCUS e IVIM quanto ao desvio padrão dos voxels de intensidade de sinal obtidos foi menor no FOCUS, mostrando uma maior homogeneidade de sinal e consequente melhor relação sinal-ruído estimada, estatisticamente significante $(p<0,001)$ (Tabela 4).

Tabela 4. Comparação entre IVIM e FOCUS quanto à RSRe

\begin{tabular}{lccc}
\hline RSRe & IVIM & FOCUS & Valor-p \\
\hline RSRe - desvio padrão & & & $<0,001$ \\
Mediana (1ํquartil; 3ํ quartil) & $113,0(78,6 ; 182,5)$ & $79,8(48,8 ; 111,8)$ & \\
Mínimo; Máximo & 36,$0 ; 771,2$ & 19,$9 ; 381,9$ & \\
\hline
\end{tabular}




\section{DISCUSSÃO}

De modo geral a sequência padrão FOCUS apresenta critérios de análise subjetiva discretamente superiores em relação à sequência teste IVIM. Essa melhor avaliação também é reforçada pelos quatro quesitos onde não foi possível a realização do teste de hipóteses (relação lesão-fundo DWI, distorção da imagem DWI, qualidade global ADC e relação lesão-fundo ADC), pois apenas a sequência IVIM foi avaliada como ruim/muito ruim (a sequência FOCUS não recebeu tal classificação em nenhum dos quesitos). No entanto, por tratar-se de análise subjetiva um fato que pode ter pesado muito é a familiaridade dos leitores com a sequência padrão e uma certa estranheza com a sequência teste.

Se analisarmos o quesito qualidade global (talvez o quesito mais importante), vemos um aspecto interessante. Especificamente na qualidade global DWI, vemos uma distribuição das avaliações muito similar, com apenas quatro casos avaliados divergentemente, e prevalência de bom/excelente para ambas as sequências, o que mostra que a sequência teste não está tão distante da sequência padrão (Tabela 2). Contudo ao olharmos especificamente para qualidade global - ADC, notamos mais uma vez a superioridade da sequência padrão, com mais casos avaliados com bom/excelente para a sequência FOCUS, e apenas a sequência IVIM recebendo avaliações ruim/muito ruim (Tabela 2). Eventualmente tal fato pode ser explicado pelos múltiplos valores de $b$ obtidos no IVIM. Como os mapas de coeficiente de difusão aparente ADC são obtidos a partir das imagens de difusão DWI, e no modelo biexponencial é necessário obtermos vários valores de $b$, em diferentes tempos, as imagens são mais suscetíveis a artefatos de movimentação da próstata decorrentes da presença/passagem de gás pelo reto.

Esse primeiro componente dos nossos resultados se assemelham em parte aos resultados obtidos no recente trabalho de Merisaari et al., ${ }^{(63)}$ que também tiveram como problemática conceitual a ausência de consenso em relação a um protocolo ideal para utilização do modelo biexponencial IVIM. Eles testaram quatro modelos de ajuste para o IVIM e, adicionalmente, quatro modelos de ajustes matemáticos para DWI, com resultados sempre superiores para 0 modelo monoexponencial. Da mesma forma nossos dados mostraram que, em geral, a sequência de difusão padrão monoexponencial FOCUS é superior ao protocolo 
biexponecial IVIM, sendo mais bem avaliada subjetivamente, e apresentando parâmetros quantitativos superiores, com menor grau de distorção das imagens e melhor RSRe. Já em outro trabalho também recente Valerio et al. ${ }^{(71)}$ mostraram aumento da especificidade e sensibilidade na detecção de cânceres na zona periféria utilizando-se do modelo biexponencial IVIM, resultado que encoraja o contínuo desenvolvimento da sequência para que possa ser incorporada na RMp. Um aspecto positivo para a sequência IVIM em nossos resultados que deve ser salientado é que a disparidade entre os números obtidos não foi tão grande; considerando-se ser uma primeira tentativa de ajuste para a sequência, novos esforços e aprimoramentos poderão torna-lá melhor.

Em relação aos parâmetros quantitativos, a sequência FOCUS também mostrou resultados superiores em relação aos da sequência IVIM, com menor distorção das imagens no eixo AP e melhor RSRe. Muito provavelmente tais resultados também podem ser explicados, ao menos em parte, pelo fato de para o IVIM serem necessárias múltiplas aquisições com diferentes valores de $b$, tornando-o mais susceptivel aos artefatos de movimentação e gerando maior heterogeneidade de sinal nas imagens adquiridas. Tal aspecto pode ser minimizado apenas com uma configuração nos pós-processamento das imagens. $O$ aparelho de RM, por configuração padrão, utiliza todos os valores de $b$ obtidos para gerar o mapa de ADC, mas isso pode ser customizado indicando ao mesmo quantos e quais valores de $b$ deverão ser usados. Isso potencialmente minimizaria os artefatos no mapa de ADC IVIM, aproximando-o em termos de qualidade ao mapa de ADC FOCUS. Essa possibilidade deverá ser objeto de trabalhos futuros.

Levando-se em consideração o enorme potencial da sequência de difusão biexponencial em tornar-se uma sequência auto-compreensiva (ou seja, ter a capacidade de, em uma única aquisição, avaliar o componente de restrição verdadeira e o componente de perfusão tecidual), com isto tornando desnecessário o uso do contraste paramagnético, desonerando o método e consequentemente tornando-o mais acessível, acreditamos que devem ser mantidos esforços para o aprimoramento desta sequência em novos estudos.

Nosso trabalho apresenta limitações. Apesar de ter uma coleta prospectiva com análise transversal, e essa ter sido realizada por dois observadores independentes, os mesmos não estavam mascarados em relação à natureza das sequências; o mascaramento não foi possível devido à peculiaridade intrínseca dos 
diferentes valores de $b$ inerentes de cada técnica de aquisição (a sequência monoexponencial é adquirida com três valores de $b$ e a biexponencial com dez valores de $b$ ), aspecto facilmente perceptível pelos examinadores. Além disso, grande parte dos resultados adveio de uma análise subjetiva, ou seja, dependente da vivência e experiência dos leitores. Soma-se o fato de sequência monoexponencial FOCUS já fazer parte há algum tempo do protocolo institucional, não sendo possível descartar viés de familiaridade dos leitores, uma vez que já estão habituados com a imagem gerada pela técnica monoexponencial, podendo o padrão de imagem da nova sequência ter causado algum grau de estranheza; contudo para minimizar e normatizar o aspecto subjetivo também foram avaliados critérios quantitativos objetivos, que se mostraram concordantes com os critérios subjetivos. 


\section{CONCLUSÃO}

1. A sequência de difusão padrão monoexponencial FOCUS obteve resultados de avaliação subjetiva e critérios quantitativos objetivos superiores em relação à sequência teste IVIM. 
6 ANEXO

Anexo 1. Protocolos difusões teste biexponencial IVIM e padrão monoexponencial FOCUS

\section{Protocolo teste biexponencial IVIM}

\begin{tabular}{ll}
\hline Parâmetro & \multicolumn{1}{c}{ Valores } \\
\hline Aparelho & 3.0 Tesla (Discovery MR 750w, GE Healthcare) \\
Tipo de de sequência de pulso & DWI single-shot spin-echo \\
Plano da varredura & oblíquo \\
Tipo de aquisição & $2 \mathrm{D}$ \\
Respiração & Livre \\
TR/TE & 4686 / 255 67,5 (otimizado ao TR) \\
Espessura do corte & $3 \mathrm{~mm}$ \\
Espaçamento & 0 \\
Número de cortes & 16 \\
Cobertura da varredura & $48 \mathrm{~mm}$ \\
Matriz & $120 \times 120$ \\
FOV (Field of view) & $200 \mathrm{~mm}$ \\
Phase encode direction & A/P \\
№ de médias & Variável \\
Sensitive encoding factor & Aquisição paralela com auto-calibração parcial generalizada \\
Valores de b (s/mm²) e número de & $0(2), 10$ (2), 30 (2), 50 (2), 80 (2), 100 (2), 200 (2), 400 (4) e \\
excitações & 1000 (8) \\
Receiver bandwidth (Hz/pixel) & $+/-250$ \\
Supressão de gordura & FOCUS \\
Tempo de aquisição & $8 m i n$ 12seg \\
\hline
\end{tabular}




\section{Protocolo padrão monoexponencial FOCUS}

\begin{tabular}{ll}
\hline Parâmetro & Valores \\
\hline Aparelho & 3.0 Tesla (Discovery MR 750w, GE Healthcare) \\
Tipo de de sequência de pulso & DWI single-shot spin-echo \\
Plano da varredura & oblíquo \\
Tipo de aquisição & $2 \mathrm{D}$ \\
Respiração & Livre \\
TR/TE & 4686 / 255 67,5 (otimizado ao TR) \\
Espessura do corte & $3 \mathrm{~mm}$ \\
Espaçamento & 0 \\
Número de cortes & 24 \\
Cobertura da varredura & $72 \mathrm{~mm}$ \\
Matriz & $120 \times 120$ \\
FOV (Field of view) & 200 \\
Phase encode direction & A/P \\
№ de médias & Variável \\
Sensitive encoding factor & Aquisição paralela com auto-calibração parcial generalizada \\
Valores de b (s/mm²) e número de & 50 (12), 1000 (16) \\
excitações & $+/-250$ \\
Receiver bandwidth (Hz/pixel) & Sim \\
Supressão de gordura & 5 min 53seg \\
Tempo de aquisição &
\end{tabular}




\section{REFERÊNCIAS}

1. Kumar V, Abbas AK, Aster JC. Robbins basic pathology. 9th ed. New York: Saunders; 2012.

2. Butler P, Mitchell A, Healy JC. Applied radiological anatomy. London: Cambridge University Press; 2012.

3. Lee $\mathrm{CH}$, Akin-Olugbade $\mathrm{O}$, Kirschenbaum A. Overview of prostate anatomy, histology, and pathology. Endocrinol Metab Clin North Am. 2011;40(3):565-75, viii-ix. Review.

4. Verma S, Rajesh A. A clinically relevant approach to imaging prostate cancer: review. AJR Am J Roentgenol. 2011;196(3 Suppl):S1-10 Quiz S11-4. Review.

5. Instituto Nacional de Cancer (INCA). Estimativas para o ano de 2016 das taxas brutas de incidência por 100 mil habitantes e do número de casos novos de câncer, segundo sexo e localização primária [Internet]. Rio de Janeiro: INCA; c1996-2018 [citado 2018 Jan 15].

Disponível em: www.inca.gov.br/estimativa/2016/tabelaestados.asp?UF=BR

6. National Cancer Institute (NIH). Cancer stat facts: prostate cancer [Internet]. Bethesda: NIH; c2018 [cited 2018 Jan 15]. Available from: http://seer.cancer.gov/statfacts/html/prost.html

7. Cancer Research UK. Prostate cancer survival statistics [Internet]. London: Cancer Research UK; c2016 [cited 19 Oct 2016]. Available from: http://www.cancerresearchuk.org/healthprofessional/cancer-statistics/statistics-by-cancer-type/prostate-cancer/survival

8. Bonekamp D, Jacobs MA, El-Khouli R, Stoianovici D, Macura KJ. Advancements in MR imaging of the prostate: from diagnosis to interventions. Radiographics. 2011;31(3):677-703.

Review.

9. Weissleder R, Wittenberg J, Harinsinghani M, Chen JW. Primer of diagnostic imaging. 5th ed. Saint Louis: Mosby; 2011.

10. Dickinson L, Ahmed HU, Allen C, Barentsz JO, Carey B, Futterer JJ, et al. Magnetic resonance imaging for the detection, localisation, and characterisation of prostate cancer: recommendations from a European consensus meeting. Eur Urol. 2011;59(4):477-94.

11. Schröder FH, Hugosson J, Roobol MJ, Tammela TL, Ciatto S, Nelen V, Kwiatkowski M, Lujan M, Lilja H, Zappa M, Denis LJ, Recker F, Berenguer A, Määttänen L, Bangma CH, Aus G, Villers A, Rebillard X, van der Kwast T, Blijenberg BG, Moss SM, de Koning HJ, Auvinen A; ERSPC Investigators. Screening and prostate-cancer mortality in a randomized European study. N Engl J Med. 2009;360(13):1320-8.

12. Carter HB, Albertsen PC, Barry MJ, Etzioni R, Freedland SJ, Greene KL, et al. Early detection of prostate cancer: AUA Guideline. Linthicum: American Urological Association; 2013.

13. Walser EM, Sze TF, Ross JR, Karamanian AA, Woodrum DA. MRI-guided prostate interventions. Am J Roentgenol. 2016;207(4):755-63.

14. Bhavsar A, Verma S. Anatomic imaging of the prostate. Biomed Res Int. 2014;2014:728539. Review. 
15. Mullins JK, Bonekamp D, Landis P, Begum H, Partin AW, Epstein Jl, et al. Multiparametric magnetic resonance imaging findings in men with low-risk prostate cancer followed using active surveillance. BJU Int. 2013;111(7):1037-45.

16. Weinreb JC, Barentsz JO, Choyke PL, Cornud F, Haider MA, Macura KJ, et al. PI-RADS Prostate imaging - reporting and data system: 2015, Version 2. Eur Urol. 2016;69(1):16-40.

17. Mussi TC, Martins T, Garcia RG, Filippi RZ, Lemos GC, Baroni RH. Are dynamic contrastenhanced images necessary for prostate cancer detection on multiparametric magnetic resonance imaging? Clin Genitourin Cancer. 2017;15(3):e447-54.

18. Iwazawa J, Mitani T, Sassa S, Ohue S. Prostate cancer detection with MRI: is dynamic contrast-enhanced imaging necessary in addition to diffusion-weighted imaging? Diagn Interv Radiol. 2011;17(3):243-8.

19. McDonald RJ, McDonald JS, Kallmes DF, Jentoft ME, Murray DL, Thielen KR, et al. Intracranial gadolinium deposition after contrast-enhanced MR imaging. Radiology. $2015 ; 275(3): 772-82$.

20. Kuhl CK, Bruhn R, Krämer N, Nebelung S, Heidenreich A, Schrading S. Abbreviated biparametric prostate MR imaging in men with elevated prostate-specific antigen. Radiology. 2017;285(2):493-505.

21. Thestrup KC, Logager V, Baslev I, Møller JM, Hansen RH, Thomsen HS. Biparametric versus multiparametric MRI in the diagnosis of prostate cancer. Acta Radiol Open. 2016;5(8):2058460116663046. Erratum in: Acta Radiol Open. 2017;6(3):2058460117699757.

22. Scialpi M, Martorana E, D'Andrea A. Standardizing biparametric MRI to simplify and improve prostate imaging reporting and data system, version 2 , in prostate cancer management. AJR Am J Roentgenol. 2016:W1-2.

23. Scialpi M, Prosperi E, D'Andrea A, Martorana E, Malaspina C, Palumbo B, et al. Biparametric versus multiparametric MRI with non-endorectal coil at $3 T$ in the detection and localization of prostate cancer. Anticancer Res. 2017;37(3):1263-1271. Erratum in: Anticancer Res. 2017;37(7):3981.

24. Scialpi M, D'Andrea A, Martorana E, Malaspina CM, Aisa MC, Napoletano M, et al. Biparametric MRI of the prostate. Turk J Urol. 2017;43(4):401-9. Review. Erratum in: Turk J Urol. 2018;44(1):91.

25. Rais-Bahrami S, Siddiqui MM, Vourganti S, Turkbey B, Rastinehad AR, Stamatakis L, et al. Diagnostic value of biparametric magnetic resonance imaging (MRI) as an adjunct to prostatespecific antigen (PSA)-based detection of prostate cancer in men without prior biopsies. BJU Int. 2015;115(3):381-8.

26. Basso L, Migone S, Prono V, Baglietto M, Rosa F, Rescinito G, et al. Comparison between multiparametric and biparametric MRI protocols for prostate cancer detection: a more efficient diagnostic protocol and a viable path toward screening? In: European Congress of Radiology; 2017 [cited 2018 Dec 10]. Vienna; Mar 1 to 5. Available from: http://dx.doi.org/10.1594/ecr2017/C-0702

27. Grover VP, Tognarelli JM, Crossey MM, Cox IJ, Taylor-Robinson SD, McPhail MJ. Magnetic resonance imaging: principles and techniques: lessons for clinicians. J Clin Exp Hepatol. 2015;5(3):246-55. Review. 
28. Malayeri AA, El Khouli RH, Zaheer A, Jacobs MA, Corona-Villalobos CP, Kamel IR, et al. Principles and applications of diffusion-weighted imaging in cancer detection, staging, and treatment follow-up. Radiographics. 2011;31(6):1773-91. Review.

29. Koh DM, Collins DJ. Diffusion-weighted MRI in the body: applications and challenges in oncology. AJR Am J Roentgenol. 2007;188(6):1622-35. Review.

30. Bammer R. Basic principles of diffusion-weighted imaging. Eur J Radiol. 2003;45(3):169-84. Review.

31. Le Bihan D, Breton E, Lallemand D, Aubin ML, Vignaud J, Laval-Jeantet M. Separation of diffusion and perfusion in intravoxel incoherent motion MR imaging. Radiology.

1988;168(2):497-505.

32. Hagmann P, Jonasson L, Maeder P, Thiran JP, Wedeen VJ, Meuli R. Understanding diffusion MR imaging techniques: from scalar diffusion-weighted imaging to diffusion tensor imaging and beyond. Radiographics. 2006;26 Suppl 1:S205-23. Review.

33. Ueno Y, Takahashi S, Ohno Y, Kitajima K, Yui M, Kassai Y, et al. Computed diffusionweighted MRI for prostate cancer detection: the influence of the combinations of $b$-values. $\mathrm{Br} \mathrm{J}$ Radiol. 2015;88(1048):20140738.

34. Renard-Penna R, Mozer P, Cornud F, Barry-Delongchamps N, Bruguière $E$, Portalez $D$, et al. Prostate imaging reporting and data system and likert scoring system: multiparametric MR imaging validation study to screen patients for initial biopsy. Radiology. 2015;275(2):458-68.

35. Luczyńska E, Heinze-Paluchowska S, Domalik A, Cwierz A, Kasperkiewicz H, Blecharz P, et al. The utility of diffusion weighted imaging (DWI) Using apparent diffusion coefficient (ADC) values in discriminating between prostate cancer and normal tissue. Pol J Radiol. 2014;79:4505.

36. Tan $\mathrm{CH}$, Wang J, Kundra V. Diffusion weighted imaging in prostate cancer. Eur Radiol. 2011;21(3):593-603. Review.

37. Le Bihan D, Breton E, Lallemand D, Grenier P, Cabanis E, Laval-Jeantet M. MR imaging of intravoxel incoherent motions: application to diffusion and perfusion in neurologic disorders.

Radiology. 1986;161(2):401-7.

38. Pavilla A, Gambarota G, Arrigo A, Mejdoubi M, Duvauferrier R, Saint-Jalmes H. Diffusional kurtosis imaging (DKI) incorporation into an intravoxel incoherent motion (IVIM) MR model to measure cerebral hypoperfusion induced by hyperventilation challenge in healthy subjects. MAGMA. 2017;30(6):545-54.

39. Federau C, Sumer S, Becce F, Maeder P, O'Brien K, Meuli R, et al. Intravoxel incoherent motion perfusion imaging in acute stroke: initial clinical experience. Neuroradiology. 2014;56(8):629-35.

40. Catanese A, Malacario F, Cirillo L, Toni F, Zenesini C, Casolino D, et al. Application of intravoxel incoherent motion (IVIM) magnetic resonance imaging in the evaluation of primitive brain tumours. Neuroradiol J. 2018;31(1):4-9.

41. Puig J, Sánchez-González J, Blasco G, Daunis-I-Estadella P, Federau C, Alberich-Bayarri Á, et al. Intravoxel incoherent motion metrics as potential biomarkers for survival in glioblastoma. PLoS One. 2016;11(7):e0158887. 
42. Eisenberger U, Thoeny HC, Binser T, Gugger M, Frey FJ, Boesch C, et al. Evaluation of renal allograft function early after transplantation with diffusion-weighted MR imaging. Eur Radiol. 2010;20(6):1374-83.

43. Luo M, Zhang L, Jiang XH, Zhang WD. Intravoxel incoherent motion: application in differentiation of hepatocellular carcinoma and focal nodular hyperplasia. Diagn Interv Radiol. 2017;23(4):263-71.

44. Shinmoto H, Tamura C, Soga S, Shiomi E, Yoshihara N, Kaji T, et al. An intravoxel incoherent motion diffusion-weighted imaging study of prostate cancer. AJR Am J Roentgenol. 2012;199(4):W496-500.

45. Döpfert J, Lemke A, Weidner A, Schad LR. Investigation of prostate cancer using diffusionweighted intravoxel incoherent motion imaging. Magn Reson Imaging. $2011 ; 29(8): 1053-8$.

46. Pang Y, Turkbey B, Bernardo M, Kruecker J, Kadoury S, Merino MJ, et al. Intravoxel incoherent motion MR imaging for prostate cancer: an evaluation of perfusion fraction and diffusion coefficient derived from different b-value combinations. Magn Reson Med. 2013;69(2):553-62.

47. Shinmoto H, Oshio K, Tanimoto A, Higuchi N, Okuda S, Kuribayashi S, et al. Biexponential apparent diffusion coefficients in prostate cancer. Magn Reson Imaging. 2009;27(3):355-9.

48. Henkelman RM. Does IVIM measure classical perfusion? Magn Reson Med. 1990;16(3):470-5.

49. Müller MF, Prasad PV, Edelman RR. Can the IVIM model be used for renal perfusion imaging? Eur J Radiol. 1998;26(3):297-303.

50. Koh DM, Collins DJ, Orton MR. Intravoxel incoherent motion in body diffusion-weighted MRI: reality and challenges. AJR Am J Roentgenol. 2011;196(6):1351-61. Review.

51. Neil JJ, Bretthorst GL. On the use of Bayesian probability theory for analysis of exponential decay data: an example taken from intravoxel incoherent motion experiments. Magn Reson Med. 1993;29(5):642-7.

52. Sakuma H, Tamagawa $\mathrm{Y}$, Kimura H, Hayashi N, Nakatsugawa S, Odori T, et al. [Intravoxel incoherent motion (IVIM) imaging using an experimental MR unit with small bore]. Nihon Igaku Hoshasen Gakkai Zasshi. 1989;49(7):941-3. Japanese.

53. Sun X, Wang H, Chen F, De Keyzer F, Yu J, Jiang Y, et al. Diffusion-weighted MRI of hepatic tumor in rats: comparison between in vivo and postmortem imaging acquisitions. $J$ Magn Reson Imaging. 2009;29(3):621-8.

54. Neil JJ, Bosch CS, Ackerman JJ. An evaluation of the sensitivity of the intravoxel incoherent motion (IVIM) method of blood flow measurement to changes in cerebral blood flow. Magn Reson Med. 1994;32(1):60-5.

55. Henkelman RM, Neil JJ, Xiang QS. A quantitative interpretation of IVIM measurements of vascular perfusion in the rat brain. Magn Reson Med. 1994;32(4):464-9.

56. Wang Z, Su MY, Najafi A, Nalcioglu O. Effect of vasodilator hydralazine on tumor microvascular random flow and blood volume as measured by intravoxel incoherent motion (IVIM) weighted MRI in conjunction with Gd-DTPA-Albumin enhanced MRI. Magn Reson Imaging. 2001;19(8):1063-72. 
57. Pickens DR 3rd, Jolgren DL, Lorenz CH, Creasy JL, Price RR. Magnetic resonance perfusion/diffusion imaging of the excised dog kidney. Invest Radiol. 1992;27(4):287-92.

58. Lemke A, Laun FB, Simon D, Stieltjes B, Schad LR. An in vivo verification of the intravoxel incoherent motion effect in diffusion-weighted imaging of the abdomen. Magn Reson Med. 2010;64(6):1580-5.

59. Yoshino N, Yamada I, Ohbayashi N, Honda E, Ida M, Kurabayashi T, et al. Salivary glands and lesions: evaluation of apparent diffusion coefficients with split-echo diffusion-weighted MR imaging--initial results. Radiology. 2001;221(3):837-42.

60. Zhang L, Murata Y, Ishida R, Ohashi I, Yoshimura R, Shibuya H. Functional evaluation with intravoxel incoherent motion echo-planar MRI in irradiated salivary glands: a correlative study with salivary gland scintigraphy. J Magn Reson Imaging. 2001;14(3):223-9.

61. Koh DM, Blackledge M, Collins DJ, Padhani AR, Wallace T, Wilton B, et al. Reproducibility and changes in the apparent diffusion coefficients of solid tumours treated with combretastatin A4 phosphate and bevacizumab in a two-centre phase I clinical trial. Eur Radiol. 2009;19(11):2728-38.

62. Lecler A, Savatovsky J, Balvay D, Zmuda M, Sadik JC, Galatoire O, et al. Repeatability of apparent diffusion coefficient and intravoxel incoherent motion parameters at 3.0 Tesla in orbital lesions. Eur Radiol. 2017;27(12):5094-103.

63. Merisaari H, Movahedi P, Perez IM, Toivonen J, Pesola M, Taimen P, et al. Fitting methods for intravoxel incoherent motion imaging of prostate cancer on region of interest level:

repeatability and gleason score prediction. Magn Reson Med. 2017;77(3):1249-64.

64. Likert R. A technique for the measurement of attitudes. Arch Psychol (Chic). 1932;140:1-55.

65. Sijbers J, Scheunders P, Bonnet N, Van Dyck D, Raman E. Quantification and improvement of the signal-to-noise ratio in a magnetic resonance image acquisition procedure. Magn Reson Imaging. 1996;14(10):1157-63.

66. Heverhagen JT. Noise measurement and estimation in MR imaging experiments. Radiology. 2007;245(3):638-9.

67. Rosenkrantz AB, Chandarana H, Pfeuffer J, Triolo MJ, Shaikh MB, Mossa DJ, et al. Zoomed echo-planar imaging using parallel transmission: impact on image quality of diffusion-weighted imaging of the prostate at 3T. Abdom Imaging. 2015;40(1):120-6.

68. Gwet KL. Handbook of inter-rater reliability: the definitive guide to measuring the extent of agreement among raters. 4th ed. Gaithersburg: Advanced Analytics;2014.

69. Altman DG. Practical statistics for medical research. London: CRC Press; 1991.

70. Stuart A. A test for homogeneity of the marginal distributions in a two-way classification. Biometrika. 1955; 42(3/4):412-6.

71. Valerio M, Zini C, Fierro D, Giura F, Colarieti A, Giuliani A, et al. 3T multiparametric MRI of the prostate: does intravoxel incoherent motion diffusion imaging have a role in the detection and stratification of prostate cancer in the peripheral zone? Eur J Radiol. 2016;85(4):790-4. 


\begin{abstract}
Purpose: To compare qualitatively and quantitatively a new biexponential diffusion sequence (IVIM) with the monoexponential standard (FOCUS) sequences on prostate MRI. Methods: This study had a prospective recruitment with cross-sectional analysis and were approved by institutional research ethics committee. Between August / 2017 and November / 2017, 70 patients who underwent multiparametric prostate magnetic resonance imaging on suspicion of prostatic neoplasia, including monoexponential (FOCUS) and biexponential (IVIM), were recruited. The images obtained by both sequences were judged by 2 independent readers regarding subjective / qualitative evaluation criteria (overall quality, noise ratio, contrast resolution / definition of the zonal anatomy, background lesion / degree of confidence in the detection of focal lesions / definition of the limits of focal lesions, definition of prostatic contours, image distortion and artifacts) and objective / quantitative (anteroposterior and laterolateral diameters of the prostates, and estimated signal-to-noise ratio), with comparison of results by statistical analysis (interobserver agreement through of the Gwet coefficient, analysis of the qualitative variables by the Stuart-Maxwell test for marginal homogeneity and analysis of the quantitative variables through Wilcoxon tests for paired samples). Results: After exclusion of four patients, the final sample consisted of 66 patients. A good/excellent agreement between observers was seen for subjective analysis criteria (except in one criteria). In the qualitative analysis good or excellent quality classification was higher for FOCUS, except in one category with evidence of significant differences for DWI global quality, DWI signal-to-noise ratio and ADC signal-to-noise ratio. In quantitative data analysis the FOCUS sequence showed lower variability of the anteroposterior diameters, meaning less distortion of the images, and better estimated signal-to-noise ratio Conclusion: Monoexponential FOCUS standard sequence was qualitatively and quantitatively better than IVIM sequence.
\end{abstract}




\section{Apêndice}

Apêndice 1. Tabelas de concordâncias interobservadores para cada parâmetro de análise qualitativa

Qualidade global - DWI

\begin{tabular}{|c|c|c|c|c|c|}
\hline \multirow{2}{*}{\multicolumn{2}{|c|}{$\begin{array}{l}\text { Qualidade global - DWI IVIM - } \\
\text { Leitor } 1\end{array}$}} & \multicolumn{3}{|c|}{ Qualidade global - DWI IVIM - Leitor 2} & \multirow[b]{2}{*}{ Total } \\
\hline & & $\begin{array}{l}\text { Ruim/Muito } \\
\text { ruim }\end{array}$ & Moderada & Bom/Excelente & \\
\hline \multirow{2}{*}{ Ruim/Muito ruim } & $\mathrm{n}$ & 0 & 0 & 0 & 0 \\
\hline & $\%$ & 0,0 & 0,0 & 0,0 & 0,0 \\
\hline \multirow{2}{*}{ Moderada } & $\mathrm{n}$ & 2 & 4 & 2 & 8 \\
\hline & $\%$ & 3,0 & 6,1 & 3,0 & 12,1 \\
\hline \multirow{2}{*}{ Bom/Excelente } & $\mathrm{n}$ & 2 & 10 & 46 & 58 \\
\hline & $\%$ & 3,0 & 15,2 & 69,7 & 87,9 \\
\hline \multirow{2}{*}{ Total } & $\mathrm{n}$ & 4 & 14 & 48 & 66 \\
\hline & $\%$ & 6,1 & 21,2 & 72,7 & 100,0 \\
\hline
\end{tabular}

Coeficiente de concordância de Gwet (IC 95\%): 0,71 (0,57; 0,85).

\begin{tabular}{lccccc}
\hline \multirow{2}{*}{$\begin{array}{l}\text { Qualidade global - DWI FOCUS - } \\
\text { Leitor } \mathbf{1}\end{array}$} & \multicolumn{2}{l}{$\begin{array}{l}\text { Qualidade global - DWI FOCUS - Leitor 2 } \\
\text { Ruim/Muito } \\
\text { ruim }\end{array}$} & Moderada & Bom/Excelente & \multirow{2}{*}{ Total } \\
\hline \multirow{2}{*}{ Ruim/Muito ruim } & $\mathrm{n}$ & 0 & 0 & 0 & 0 \\
\hline \multirow{2}{*}{ Moderada } & $\%$ & 0,0 & 0,0 & 0,0 & 0,0 \\
\hline \multirow{2}{*}{ Bom/Excelente } & $\mathrm{n}$ & 1 & 3 & 0 & 4 \\
& $\%$ & 1,5 & 4,5 & 0,0 & 6,1 \\
\hline \multirow{2}{*}{ Total } & $\mathrm{n}$ & 3 & 8 & 51 & 62 \\
& $\mathrm{n}$ & 4,5 & 12,1 & 77,3 & 93,9 \\
\hline
\end{tabular}

Coeficiente de concordância de Gwet (IC 95\%): 0,79 (0,67; 0,91).

Relação sinal-ruído - DWI

\begin{tabular}{|c|c|c|c|c|c|}
\hline \multirow{2}{*}{\multicolumn{2}{|c|}{$\begin{array}{l}\text { Relação sinal-ruído - DWI IVIM - } \\
\text { Leitor } 1\end{array}$}} & \multicolumn{3}{|c|}{ Relação sinal-ruído - DWI IVIM - Leitor 2} & \multirow[b]{2}{*}{ Total } \\
\hline & & $\begin{array}{l}\text { Ruim/Muito } \\
\text { ruim }\end{array}$ & Moderada & Bom/Excelente & \\
\hline \multirow{2}{*}{ Ruim/Muito ruim } & $\mathrm{n}$ & 0 & 0 & 0 & 0 \\
\hline & $\%$ & 0,0 & 0,0 & 0,0 & 0,0 \\
\hline \multirow{2}{*}{ Moderada } & $\mathrm{n}$ & 0 & 2 & 13 & 15 \\
\hline & $\%$ & 0,0 & 3,0 & 19,7 & 22,7 \\
\hline \multirow{2}{*}{ Bom/Excelente } & $\mathrm{n}$ & 0 & 3 & 48 & 51 \\
\hline & $\%$ & 0,0 & 4,5 & 72,7 & 77,3 \\
\hline \multirow{2}{*}{ Total } & $\mathrm{n}$ & 0 & 5 & 61 & 66 \\
\hline & $\%$ & 0,0 & 7,6 & 92,4 & 100,0 \\
\hline
\end{tabular}

Coeficiente de concordância de Gwet (IC 95\%): 0,67 (0,50; 0,85). 


\begin{tabular}{|c|c|c|c|c|c|}
\hline \multirow{2}{*}{\multicolumn{2}{|c|}{$\begin{array}{l}\text { Relação sinal-ruído - DWI FOCUS } \\
\text { - Leitor } 1\end{array}$}} & \multicolumn{3}{|c|}{ Relação sinal-ruído - DWI FOCUS - Leitor 2} & \multirow[b]{2}{*}{ Total } \\
\hline & & $\begin{array}{l}\text { Ruim/Muito } \\
\text { ruim }\end{array}$ & Moderada & Bom/Excelente & \\
\hline \multirow{2}{*}{ Ruim/Muito ruim } & $\mathrm{n}$ & 0 & 0 & 0 & 0 \\
\hline & $\%$ & 0,0 & 0,0 & 0,0 & 0,0 \\
\hline \multirow{2}{*}{ Moderada } & $\mathrm{n}$ & 0 & 1 & 5 & 6 \\
\hline & $\%$ & 0,0 & 1,5 & 7,6 & 9,1 \\
\hline \multirow{2}{*}{ Bom/Excelente } & $\mathrm{n}$ & 0 & 1 & 59 & 60 \\
\hline & $\%$ & 0,0 & 1,5 & 89,4 & 90,9 \\
\hline \multirow{2}{*}{ Total } & $\mathrm{n}$ & 0 & 2 & 64 & 66 \\
\hline & $\%$ & 0,0 & 3,0 & 97,0 & 100,0 \\
\hline
\end{tabular}

Coeficiente de concordância de Gwet (IC 95\%): 0,90 (0,81; 0,98).

Resolução de contraste / Definição da anatomia zonal - DWI

\section{Resolução de contraste /}

Definição da anatomia zonal DWI IVIM - Leitor 1

Ruim/Muito ruim
Moderada
Bom/Excelente
Total

Resolução de contraste / Definição da anatomia zonal -

DWI IVIM - Leitor 2

Total

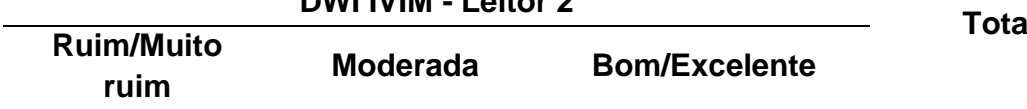

\begin{tabular}{ccccc}
$\mathrm{n}$ & 0 & 0 & 0 & 0 \\
$\%$ & 0,0 & 0,0 & 0,0 & 0,0 \\
\hline $\mathrm{n}$ & 0 & 4 & 6 & 10 \\
$\%$ & 0,0 & 6,1 & 9,1 & 15,2 \\
\hline $\mathrm{n}$ & 0 & 1 & 55 & 56 \\
$\%$ & 0,0 & 1,5 & 83,3 & 84,8 \\
\hline $\mathrm{n}$ & 0 & 5 & 61 & 66 \\
$\%$ & 0,0 & 7,6 & 92,4 & 100,0 \\
\hline
\end{tabular}

Coeficiente de concordância de Gwet (IC 95\%): 0,87 (0,76; 0,97).

Resolução de contraste /

Definição da anatomia zonal -

DWI FOCUS - Leitor 1

Resolução de contraste / Definição da anatomia zonal DWI FOCUS - Leitor 2

\begin{tabular}{|c|c|c|c|c|c|}
\hline \multirow{2}{*}{\multicolumn{2}{|c|}{$\begin{array}{l}\text { Definição da anatomia zonal - } \\
\text { DWI FOCUS - Leitor } 1\end{array}$}} & \multicolumn{3}{|c|}{ DWI FOCUS - Leitor 2} & \multirow{2}{*}{ Total } \\
\hline & & $\begin{array}{l}\text { Ruim/Muito } \\
\text { ruim }\end{array}$ & Moderada & Bom/Excelente & \\
\hline \multirow{2}{*}{ Ruim/Muito ruim } & $\mathrm{n}$ & 0 & 0 & 0 & 0 \\
\hline & $\%$ & 0,0 & 0,0 & 0,0 & 0,0 \\
\hline \multirow{2}{*}{ Moderada } & $\mathrm{n}$ & 0 & 3 & 2 & 5 \\
\hline & $\%$ & 0,0 & 4,5 & 3,0 & 7,6 \\
\hline \multirow{2}{*}{ Bom/Excelente } & $\mathrm{n}$ & 0 & 1 & 60 & 61 \\
\hline & $\%$ & 0,0 & 1,5 & 90,9 & 92,4 \\
\hline \multirow{2}{*}{ Total } & $\mathrm{n}$ & 0 & 4 & 62 & 66 \\
\hline & $\%$ & 0,0 & 6,1 & 93,9 & 100,0 \\
\hline
\end{tabular}

Coeficiente de concordância de Gwet (IC 95\%): 0,95 (0,89; 1,01). 
Relação Lesão Fundo / Grau de confiança na detecção de lesões focais / Definição dos limites de lesões focais - DWI

\begin{tabular}{|c|c|c|c|c|c|c|}
\hline \multirow{2}{*}{\multicolumn{2}{|c|}{$\begin{array}{l}\text { Relação Lesão Fundo / Grau } \\
\text { de confiança na detecção de } \\
\text { lesões focais / Definição dos } \\
\text { limites de lesões focais - DWI } \\
\text { IVIM - Leitor } 1\end{array}$}} & \multicolumn{4}{|c|}{$\begin{array}{l}\text { Relação Lesão Fundo / Grau de confiança na detecção de lesões } \\
\text { focais / Definição dos limites de lesões focais - DWI IVIM - Leitor } 2\end{array}$} & \multirow[b]{2}{*}{ Total } \\
\hline & & Não se aplica & $\begin{array}{c}\text { Ruim/Muito } \\
\text { ruim }\end{array}$ & Moderada & Bom/Excelente & \\
\hline \multirow{2}{*}{ Não se aplica } & $\mathrm{n}$ & 43 & 0 & 5 & 4 & 52 \\
\hline & $\%$ & 65,2 & 0,0 & 7,6 & 6,1 & 78,8 \\
\hline \multirow{2}{*}{ Ruim/Muito ruim } & $\mathrm{n}$ & 1 & 0 & 0 & 0 & 1 \\
\hline & $\%$ & 1,5 & 0,0 & 0,0 & 0,0 & 1,5 \\
\hline \multirow{2}{*}{ Moderada } & $\mathrm{n}$ & 3 & 0 & 2 & 3 & 8 \\
\hline & $\%$ & 4,5 & 0,0 & 3,0 & 4,5 & 12,1 \\
\hline \multirow{2}{*}{ Bom/Excelente } & $\mathrm{n}$ & 0 & 1 & 1 & 3 & 5 \\
\hline & $\%$ & 0,0 & 1,5 & 1,5 & 4,5 & 7,6 \\
\hline \multirow{2}{*}{ Total } & $\mathrm{n}$ & 47 & 1 & 8 & 10 & 66 \\
\hline & $\%$ & 71,2 & 1,5 & 12,1 & 15,2 & 100,0 \\
\hline
\end{tabular}

Coeficiente de concordância de Gwet (IC 95\%): 0,68 (0,55; 0,82).

\begin{tabular}{|c|c|c|c|c|c|c|}
\hline \multirow{2}{*}{\multicolumn{2}{|c|}{$\begin{array}{l}\text { Relação Lesão Fundo / Grau } \\
\text { de confiança na detecção de } \\
\text { lesões focais / Definição dos } \\
\text { limites de lesões focais - DWI } \\
\text { FOCUS - Leitor } 1\end{array}$}} & \multicolumn{4}{|c|}{$\begin{array}{c}\text { Relação Lesão Fundo / Grau de confiança na detecção de lesões } \\
\text { focais / Definição dos limites de lesões focais - DWI FOCUS - } \\
\text { Leitor } 2\end{array}$} & \multirow[t]{2}{*}{ Total } \\
\hline & & Não se aplica & $\begin{array}{l}\text { Ruim/Muito } \\
\text { ruim }\end{array}$ & Moderada & Bom/Excelente & \\
\hline \multirow{2}{*}{ Não se aplica } & $\mathrm{n}$ & 42 & 0 & 5 & 4 & 51 \\
\hline & $\%$ & 63,6 & 0,0 & 7,6 & 6,1 & 77,3 \\
\hline \multirow{2}{*}{ Ruim/Muito ruim } & $\mathrm{n}$ & 0 & 0 & 0 & 0 & 0 \\
\hline & $\%$ & 0,0 & 0,0 & 0,0 & 0,0 & 0,0 \\
\hline \multirow{2}{*}{ Moderada } & $\mathrm{n}$ & 2 & 0 & 0 & 1 & 3 \\
\hline & $\%$ & 3,0 & 0,0 & 0,0 & 1,5 & 4,5 \\
\hline \multirow{2}{*}{ Bom/Excelente } & $\mathrm{n}$ & 2 & 2 & 1 & 7 & 12 \\
\hline & $\%$ & 3,0 & 3,0 & 1,5 & 10,6 & 18,2 \\
\hline \multirow{2}{*}{ Total } & $\mathrm{n}$ & 46 & 2 & 6 & 12 & 66 \\
\hline & $\%$ & 69,7 & 3,0 & 9,1 & 18,2 & 100,0 \\
\hline
\end{tabular}

Coeficiente de concordância de Gwet (IC 95\%): 0,70 (0,57; 0,83).

\section{Definição dos contornos prostáticos - DWI}

\begin{tabular}{|c|c|c|c|c|c|}
\hline \multirow{3}{*}{\multicolumn{2}{|c|}{$\begin{array}{l}\text { Definição dos contornos } \\
\text { prostáticos - DWI IVIM - Leitor } 1\end{array}$}} & \multicolumn{3}{|c|}{ Definição dos contornos prostáticos - DWI IVIM - Leitor } & \multirow{3}{*}{ Total } \\
\hline & & \multirow{3}{*}{$\begin{array}{c}\text { Ruim/Muito } \\
\text { ruim }\end{array}$} & 2 & \multirow[b]{2}{*}{ Bom/Excelente } & \\
\hline & & & Moderada & & \\
\hline \multirow{2}{*}{ Ruim/Muito ruim } & $\mathrm{n}$ & & 0 & 0 & 0 \\
\hline & $\%$ & 0,0 & 0,0 & 0,0 & 0,0 \\
\hline \multirow{2}{*}{ Moderada } & $\mathrm{n}$ & 1 & 2 & 3 & 6 \\
\hline & $\%$ & 1,5 & 3,0 & 4,5 & 9,1 \\
\hline \multirow{2}{*}{ Bom/Excelente } & $\mathrm{n}$ & 1 & 16 & 43 & 60 \\
\hline & $\%$ & 1,5 & 24,2 & 65,2 & 90,9 \\
\hline \multirow{2}{*}{ Total } & $\mathrm{n}$ & 2 & 18 & 46 & 66 \\
\hline & $\%$ & 3,0 & 27,3 & 69,7 & 100,0 \\
\hline
\end{tabular}

Coeficiente de concordância de Gwet (IC 95\%): 0,62 (0,47; 0,77). 


\begin{tabular}{|c|c|c|c|c|c|}
\hline \multirow{2}{*}{\multicolumn{2}{|c|}{$\begin{array}{l}\text { Definição dos contornos } \\
\text { prostáticos - DWI FOCUS - } \\
\text { Leitor } 1\end{array}$}} & \multicolumn{3}{|c|}{ Definição dos contornos prostáticos - DWI FOCUS - Leitor 2} & \multirow{2}{*}{ Total } \\
\hline & & Ruim/Muito ruim & Moderada & Bom/Excelente & \\
\hline \multirow{2}{*}{ Ruim/Muito ruim } & $\mathrm{n}$ & 0 & 0 & 0 & 0 \\
\hline & $\%$ & 0,0 & 0,0 & 0,0 & 0,0 \\
\hline \multirow{2}{*}{ Moderada } & $\mathrm{n}$ & 1 & 3 & 4 & 8 \\
\hline & $\%$ & 1,5 & 4,5 & 6,1 & 12,1 \\
\hline \multirow{2}{*}{ Bom/Excelente } & $\mathrm{n}$ & 1 & 8 & 49 & 58 \\
\hline & $\%$ & 1,5 & 12,1 & 74,2 & 87,9 \\
\hline \multirow{2}{*}{ Total } & $\mathrm{n}$ & 2 & 11 & 53 & 66 \\
\hline & $\%$ & 3,0 & 16,7 & 80,3 & 100,0 \\
\hline
\end{tabular}

Coeficiente de concordância de Gwet (IC 95\%): 0,75 (0,63; 0,88).

\section{Distorção da imagem - DWI}

\begin{tabular}{|c|c|c|c|c|c|}
\hline \multirow{2}{*}{\multicolumn{2}{|c|}{$\begin{array}{l}\text { Distorção da imagem - DWI IVIM - } \\
\text { Leitor } 1\end{array}$}} & \multicolumn{3}{|c|}{ Distorção da imagem - DWI IVIM - Leitor 2} & \multirow[b]{2}{*}{ Total } \\
\hline & & $\begin{array}{l}\text { Ruim/Muito } \\
\text { ruim }\end{array}$ & Moderada & Bom/Excelente & \\
\hline \multirow{2}{*}{ Ruim/Muito ruim } & $\mathrm{n}$ & 0 & 1 & 1 & 2 \\
\hline & $\%$ & 0,0 & 1,5 & 1,5 & 3,0 \\
\hline \multirow{2}{*}{ Moderada } & $\mathrm{n}$ & 2 & 3 & 4 & 9 \\
\hline & $\%$ & 3,0 & 4,5 & 6,1 & 13,6 \\
\hline \multirow{2}{*}{ Bom/Excelente } & $\mathrm{n}$ & 0 & 3 & 52 & 55 \\
\hline & $\%$ & 0,0 & 4,5 & 78,8 & 83,3 \\
\hline \multirow{2}{*}{ Total } & $\mathrm{n}$ & 2 & 7 & 57 & 66 \\
\hline & $\%$ & 3,0 & 10,6 & 86,4 & 100,0 \\
\hline
\end{tabular}

Coeficiente de concordância de Gwet (IC 95\%): 0,81 (0,69; 0,92).

\begin{tabular}{|c|c|c|c|c|c|}
\hline \multirow{2}{*}{\multicolumn{2}{|c|}{$\begin{array}{l}\text { Distorção da imagem - DWI } \\
\text { FOCUS - Leitor } 1\end{array}$}} & \multicolumn{3}{|c|}{ Distorção da imagem - DWI FOCUS - Leitor 2} & \multirow[b]{2}{*}{ Total } \\
\hline & & $\begin{array}{l}\text { Ruim/Muito } \\
\text { ruim }\end{array}$ & Moderada & Bom/Excelente & \\
\hline \multirow{2}{*}{ Ruim/Muito ruim } & $\mathrm{n}$ & 0 & 0 & 0 & 0 \\
\hline & $\%$ & 0,0 & 0,0 & 0,0 & 0,0 \\
\hline \multirow{2}{*}{ Moderada } & $\mathrm{n}$ & 2 & 4 & 4 & 10 \\
\hline & $\%$ & 3,0 & 6,1 & 6,1 & 15,2 \\
\hline \multirow{2}{*}{ Bom/Excelente } & $\mathrm{n}$ & 0 & 2 & 54 & 56 \\
\hline & $\%$ & 0,0 & 3,0 & 81,8 & 84,8 \\
\hline \multirow{2}{*}{ Total } & $\mathrm{n}$ & 2 & 6 & 58 & 66 \\
\hline & $\%$ & 3,0 & 9,1 & 87,9 & 100,0 \\
\hline
\end{tabular}

Coeficiente de concordância de Gwet (IC 95\%): 0,86 (0,77; 0,96).

\section{Artefatos - DWI}

\begin{tabular}{|c|c|c|c|c|c|}
\hline \multirow{2}{*}{\multicolumn{2}{|c|}{ Artefatos - DWI IVIM - Leitor 1}} & \multicolumn{3}{|c|}{ Artefatos - DWI IVIM - Leitor 2} & \multirow{3}{*}{$\begin{array}{c}\text { Total } \\
1\end{array}$} \\
\hline & & \multirow{2}{*}{$\begin{array}{c}\begin{array}{c}\text { Ruim/Muito } \\
\text { ruim }\end{array} \\
1\end{array}$} & \multirow{2}{*}{$\begin{array}{c}\text { Moderada } \\
0\end{array}$} & \multirow{2}{*}{$\begin{array}{c}\text { Bom/Excelente } \\
0\end{array}$} & \\
\hline \multirow{2}{*}{ Ruim/Muito ruim } & $\mathrm{n}$ & & & & \\
\hline & $\%$ & 1,5 & 0,0 & 0,0 & 1,5 \\
\hline \multirow{2}{*}{ Moderada } & $\mathrm{n}$ & 3 & 2 & 5 & 10 \\
\hline & $\%$ & 4,5 & 3,0 & 7,6 & 15,2 \\
\hline \multirow{2}{*}{ Bom/Excelente } & $\mathrm{n}$ & 0 & 11 & 44 & 55 \\
\hline & $\%$ & 0,0 & 16,7 & 66,7 & 83,3 \\
\hline \multirow{2}{*}{ Total } & $\mathrm{n}$ & 4 & 13 & 49 & 66 \\
\hline & $\%$ & 6,1 & 19,7 & 74,2 & 100,0 \\
\hline
\end{tabular}

Coeficiente de concordância de Gwet (IC 95\%): 0,65 (0,50; 0,80). 


\begin{tabular}{|c|c|c|c|c|c|}
\hline \multirow{2}{*}{\multicolumn{2}{|c|}{ Artefatos - DWI FOCUS - Leitor 1}} & \multicolumn{3}{|c|}{ Artefatos - DWI FOCUS - Leitor 2} & \multirow{3}{*}{$\begin{array}{c}\text { Total } \\
1\end{array}$} \\
\hline & & \multirow{2}{*}{$\begin{array}{c}\text { Ruim/Muito } \\
\text { ruim }\end{array}$} & \multirow{2}{*}{$\begin{array}{c}\text { Moderada } \\
0\end{array}$} & \multirow{2}{*}{$\begin{array}{c}\text { Bom/Excelente } \\
0\end{array}$} & \\
\hline \multirow{2}{*}{ Ruim/Muito ruim } & $\mathrm{n}$ & & & & \\
\hline & $\%$ & 1,5 & 0,0 & 0,0 & 1,5 \\
\hline \multirow{2}{*}{ Moderada } & $\mathrm{n}$ & 3 & 2 & 4 & 9 \\
\hline & $\%$ & 4,5 & 3,0 & 6,1 & 13,6 \\
\hline \multirow{2}{*}{ Bom/Excelente } & $\mathrm{n}$ & 0 & 8 & 48 & 56 \\
\hline & $\%$ & 0,0 & 12,1 & 72,7 & 84,8 \\
\hline \multirow{2}{*}{ Total } & $\mathrm{n}$ & 4 & 10 & 52 & 66 \\
\hline & $\%$ & 6,1 & 15,2 & 78,8 & 100,0 \\
\hline
\end{tabular}

Coeficiente de concordância de Gwet (IC 95\%): 0,73 (0,60; 0,87).

Qualidade global - ADC

\begin{tabular}{|c|c|c|c|c|c|}
\hline \multirow{2}{*}{\multicolumn{2}{|c|}{$\begin{array}{l}\text { Qualidade global - ADC IVIM - } \\
\text { Leitor } 1\end{array}$}} & \multicolumn{3}{|c|}{ Qualidade global - ADC IVIM - Leitor 2} & \multirow[b]{2}{*}{ Total } \\
\hline & & $\begin{array}{l}\text { Ruim/Muito } \\
\text { ruim }\end{array}$ & Moderada & Bom/Excelente & \\
\hline \multirow{2}{*}{ Ruim/Muito ruim } & $\mathrm{n}$ & 1 & 1 & 0 & 2 \\
\hline & $\%$ & 1,5 & 1,5 & 0,0 & 3,0 \\
\hline \multirow{2}{*}{ Moderada } & $\mathrm{n}$ & 3 & 9 & 5 & 17 \\
\hline & $\%$ & 4,5 & 13,6 & 7,6 & 25,8 \\
\hline \multirow{2}{*}{ Bom/Excelente } & $\mathrm{n}$ & 1 & 5 & 41 & 47 \\
\hline & $\%$ & 1,5 & 7,6 & 62,1 & 71,2 \\
\hline \multirow{2}{*}{ Total } & $\mathrm{n}$ & 5 & 15 & 46 & 66 \\
\hline & $\%$ & 7,6 & 22,7 & 69,7 & 100,0 \\
\hline
\end{tabular}

Coeficiente de concordância de Gwet (IC 95\%): 0,71 (0,57; 0,85).

\begin{tabular}{lccccc}
\hline \multirow{2}{*}{$\begin{array}{l}\text { Qualidade global - ADC FOCUS - } \\
\text { Leitor 1 }\end{array}$} & \multicolumn{2}{c}{ Qualidade global - ADC FOCUS - Leitor 2 } & Total \\
\cline { 2 - 5 } Ruim/Muito & ruim & Moderada & Bom/Excelente & \\
\hline \multirow{2}{*}{ Ruim/Muito ruim } & $\mathrm{n}$ & 0 & 0 & 0 & 0 \\
\hline \multirow{2}{*}{ Moderada } & $\%$ & 0,0 & 0,0 & 0,0 & 0,0 \\
\hline \multirow{2}{*}{ Bom/Excelente } & $\mathrm{n}$ & 2 & 2 & 2 & 6 \\
& $\%$ & 3,0 & 3,0 & 3,0 & 9,1 \\
\hline \multirow{2}{*}{ Total } & $\mathrm{n}$ & 2 & 4 & 54 & 60 \\
& $\mathrm{n}$ & 3,0 & 6,1 & 81,8 & 90,9 \\
\hline
\end{tabular}

Coeficiente de concordância de Gwet (IC 95\%): 0,83 (0,72; 0,94).

\section{Relação sinal-ruído - ADC}

\begin{tabular}{lccccc}
\hline \multirow{2}{*}{$\begin{array}{l}\text { Relação sinal-ruído - ADC IVIM - } \\
\text { Leitor 1 }\end{array}$} & \multicolumn{2}{c}{$\begin{array}{c}\text { Relação sinal-ruído - ADC IVIM - Leitor 2 } \\
\text { Ruim/Muito } \\
\text { ruim }\end{array}$} & Moderada & Bom/Excelente & \multirow{2}{*}{ Total } \\
\hline \multirow{2}{*}{ Ruim/Muito ruim } & $\mathrm{n}$ & 0 & 3 & 4 & 7 \\
\hline \multirow{2}{*}{ Moderada } & $\%$ & 0,0 & 4,5 & 6,1 & 10,6 \\
\hline \multirow{2}{*}{ Bom/Excelente } & $\mathrm{n}$ & 1 & 8 & 24 & 33 \\
& $\%$ & 1,5 & 12,1 & 36,4 & 50,0 \\
\hline \multirow{2}{*}{ Total } & $\mathrm{n}$ & 0 & 1 & 37,9 & 26 \\
& $\mathrm{n}$ & 0,0 & 1,5 & 53 & 39,4 \\
\hline
\end{tabular}

Coeficiente de concordância de Gwet (IC 95\%): 0,32 (0,14; 0,50). 


\begin{tabular}{lccccc}
\hline \multirow{2}{*}{$\begin{array}{l}\text { Relação sinal-ruído - ADC } \\
\text { FOCUS - Leitor 1 }\end{array}$} & & \multicolumn{2}{c}{ Relação sinal-ruído - ADC FOCUS - Leitor 2 } & Total \\
\cline { 2 - 5 } & $\mathrm{n}$ & $\begin{array}{c}\text { Ruim/Muito } \\
\text { ruim }\end{array}$ & Moderada & Bom/Excelente & 1 \\
\hline \multirow{2}{*}{ Ruim/Muito ruim } & $\%$ & 0,0 & 0 & 1 & 1,5 \\
\hline \multirow{2}{*}{ Moderada } & $\mathrm{n}$ & 0 & 0,0 & 3 & 3 \\
& $\%$ & 0,0 & 0 & 4,5 & 4,5 \\
\hline \multirow{2}{*}{ Bom/Excelente } & $\mathrm{n}$ & 0 & 0,0 & 60 & 62 \\
& $\%$ & 0,0 & 3,0 & 90,9 & 93,9 \\
\multirow{2}{*}{ Total } & $\mathrm{n}$ & 0 & 2 & 64 & 66 \\
& $\%$ & 0,0 & 3,0 & 97,0 & 100,0 \\
\hline
\end{tabular}

Coeficiente de concordância de Gwet (IC 95\%): 0,90 (0,83; 0,98).

Resolução de contraste / Definição da anatomia zonal - ADC

Resolução de contraste / Resolução de contraste / Definição da anatomia zonal -

Definição da anatomia zonal ADC IVIM - Leitor 1 ADC IVIM - Leitor 2

Total

\begin{tabular}{lccccc}
\multicolumn{7}{c}{ ruim } & 0 & 2 & 2 \\
Ruim/Muito ruim & $\mathrm{n}$ & 0 & 0,0 & 3,0 & 3,0 \\
\hline \multirow{2}{*}{ Moderada } & $\%$ & 0,0 & 1 & 18 & 19 \\
& $\mathrm{n}$ & 0 & 1,5 & 27,3 & 28,8 \\
\hline \multirow{2}{*}{ Bom/Excelente } & $\%$ & 0,0 & 2 & 43 & 45 \\
& $\mathrm{n}$ & 0 & 3,0 & 65,2 & 68,2 \\
\hline \multirow{2}{*}{ Total } & $\mathrm{n}$ & 0,0 & 3 & 63 & 66 \\
& $\%$ & 0,0 & 4,5 & 95,5 & 100,0 \\
\hline
\end{tabular}

Coeficiente de concordância de Gwet (IC 95\%): 0,61 (0,45; 0,76).

\begin{tabular}{|c|c|c|c|c|c|}
\hline \multirow{2}{*}{\multicolumn{2}{|c|}{$\begin{array}{l}\text { Resolução de contraste / } \\
\text { Definição da anatomia zonal - } \\
\text { ADC FOCUS - Leitor } 1\end{array}$}} & \multicolumn{3}{|c|}{$\begin{array}{l}\text { Resolução de contraste / Definição da anatomia zonal - } \\
\text { ADC FOCUS - Leitor } 2\end{array}$} & \multirow{2}{*}{ Total } \\
\hline & & $\begin{array}{l}\text { Ruim/Muito } \\
\text { ruim }\end{array}$ & Moderada & Bom/Excelente & \\
\hline \multirow{2}{*}{ Ruim/Muito ruim } & $\mathrm{n}$ & 0 & 0 & 1 & 1 \\
\hline & $\%$ & 0,0 & 0,0 & 1,5 & 1,5 \\
\hline \multirow{2}{*}{ Moderada } & $\mathrm{n}$ & 0 & 0 & 13 & 13 \\
\hline & $\%$ & 0,0 & 0,0 & 19,7 & 19,7 \\
\hline \multirow{2}{*}{ Bom/Excelente } & $\mathrm{n}$ & 0 & 2 & 50 & 52 \\
\hline & $\%$ & 0,0 & 3,0 & 75,8 & 78,8 \\
\hline \multirow{2}{*}{ Total } & $\mathrm{n}$ & 0 & 2 & 64 & 66 \\
\hline & $\%$ & 0,0 & 3,0 & 97,0 & 100,0 \\
\hline
\end{tabular}

Coeficiente de concordância de Gwet (IC 95\%): 0,73 (0,60; 0,86). 
Relação Lesão Fundo / Grau de confiança na detecção de lesões focais / Definição dos limites de lesões focais - ADC

\begin{tabular}{|c|c|c|c|c|c|c|}
\hline \multirow{2}{*}{\multicolumn{2}{|c|}{$\begin{array}{l}\text { Relação Lesão Fundo / Grau } \\
\text { de confiança na detecção de } \\
\text { lesões focais / Definição dos } \\
\text { limites de lesões focais - } \\
\text { ADC IVIM - Leitor } 1\end{array}$}} & \multicolumn{4}{|c|}{$\begin{array}{l}\text { Relação Lesão Fundo / Grau de confiança na detecção de lesões } \\
\text { focais / Definição dos limites de lesões focais - ADC IVIM - Leitor } 2\end{array}$} & \multirow[b]{2}{*}{ Total } \\
\hline & & Não se aplica & $\begin{array}{l}\text { Ruim/Muito } \\
\text { ruim }\end{array}$ & Moderada & Bom/Excelente & \\
\hline \multirow{2}{*}{ Não se aplica } & $\mathrm{n}$ & 42 & 0 & 6 & 4 & 52 \\
\hline & $\%$ & 63,6 & 0,0 & 9,1 & 6,1 & 78,8 \\
\hline \multirow{2}{*}{ Ruim/Muito ruim } & $\mathrm{n}$ & 0 & 0 & 1 & 0 & 1 \\
\hline & $\%$ & 0,0 & 0,0 & 1,5 & 0,0 & 1,5 \\
\hline \multirow{2}{*}{ Moderada } & $\mathrm{n}$ & 2 & 0 & 0 & 2 & 4 \\
\hline & $\%$ & 3,0 & 0,0 & 0,0 & 3,0 & 6,1 \\
\hline \multirow{2}{*}{ Bom/Excelente } & $\mathrm{n}$ & 3 & 0 & 1 & 5 & 9 \\
\hline & $\%$ & 4,5 & 0,0 & 1,5 & 7,6 & 13,6 \\
\hline \multirow{2}{*}{ Total } & $\mathrm{n}$ & 47 & 0 & 8 & 11 & 66 \\
\hline & $\%$ & 71,2 & 0,0 & 12,1 & 16,7 & 100,0 \\
\hline
\end{tabular}

Coeficiente de concordância de Gwet (IC 95\%): 0,67 (0,53; 0,81).

\begin{tabular}{|c|c|c|c|c|c|c|}
\hline \multirow{2}{*}{\multicolumn{2}{|c|}{$\begin{array}{l}\text { Relação Lesão Fundo / } \\
\text { Grau de confiança na } \\
\text { detecção de lesões focais / } \\
\text { Definição dos limites de } \\
\text { lesões focais - ADC FOCUS } \\
\text { - Leitor } 1\end{array}$}} & \multicolumn{4}{|c|}{$\begin{array}{c}\text { Relação Lesão Fundo / Grau de confiança na detecção de lesões } \\
\text { focais / Definição dos limites de lesões focais - ADC FOCUS - } \\
\text { Leitor } 2\end{array}$} & \multirow{2}{*}{ Total } \\
\hline & & $\begin{array}{c}\text { Não se } \\
\text { aplica }\end{array}$ & Ruim/Muito ruim & Moderada & Bom/Excelente & \\
\hline \multirow{2}{*}{ Não se aplica } & $\mathrm{n}$ & 41 & 0 & 1 & 8 & 50 \\
\hline & $\%$ & 62,1 & 0,0 & 1,5 & 12,1 & 75,8 \\
\hline \multirow{2}{*}{ Ruim/Muito ruim } & $\mathrm{n}$ & 0 & 0 & 0 & 0 & 0 \\
\hline & $\%$ & 0,0 & 0,0 & 0,0 & 0,0 & 0,0 \\
\hline \multirow{2}{*}{ Moderada } & $\mathrm{n}$ & 2 & 0 & 0 & 2 & 4 \\
\hline & $\%$ & 3,0 & 0,0 & 0,0 & 3,0 & 6,1 \\
\hline \multirow{2}{*}{ Bom/Excelente } & $\mathrm{n}$ & 3 & 0 & 3 & 6 & 12 \\
\hline & $\%$ & 4,5 & 0,0 & 4,5 & 9,1 & 18,2 \\
\hline \multirow{2}{*}{ Total } & $\mathrm{n}$ & 46 & 0 & 4 & 16 & 66 \\
\hline & $\%$ & 69,7 & 0,0 & 6,1 & 24,2 & 100,0 \\
\hline
\end{tabular}

Coeficiente de concordância de Gwet (IC 95\%): 0,64 (0,48; 0,79).

\section{Definição dos contornos prostáticos - ADC}

\begin{tabular}{lccccc}
\hline \multirow{2}{*}{$\begin{array}{l}\text { Definição dos contornos } \\
\text { prostáticos - ADC IVIM - Leitor } \mathbf{1}\end{array}$} & \multicolumn{2}{l}{ Definição dos contornos prostáticos - ADC IVIM - Leitor } & \multirow{2}{*}{ Total } \\
\cline { 2 - 5 } & & $\begin{array}{c}\text { Ruim/Muito } \\
\text { ruim }\end{array}$ & Moderada & Bom/Excelente & \\
\hline \multirow{2}{*}{ Ruim/Muito ruim } & $\mathrm{n}$ & 0 & 1 & 0 & 1 \\
& $\%$ & 0,0 & 1,5 & 0,0 & 1,5 \\
\hline \multirow{2}{*}{ Moderada } & $\mathrm{n}$ & 2 & 4 & 3 & 9 \\
& $\%$ & 3,0 & 6,1 & 4,5 & 13,6 \\
\hline \multirow{2}{*}{ Bom/Excelente } & $\mathrm{n}$ & 1 & 8 & 47 & 56 \\
\hline \multirow{2}{*}{ Total } & $\%$ & 1,5 & 12,1 & 71,2 & 84,8 \\
\hline
\end{tabular}

Coeficiente de concordância de Gwet (IC 95\%): 0,73 (0,59; 0,86). 


\begin{tabular}{|c|c|c|c|c|c|}
\hline \multirow{2}{*}{\multicolumn{2}{|c|}{$\begin{array}{l}\text { Definição dos contornos } \\
\text { prostáticos - ADC FOCUS - } \\
\text { Leitor } 1\end{array}$}} & \multicolumn{3}{|c|}{$\begin{array}{c}\text { Definição dos contornos prostáticos - ADC FOCUS - } \\
\text { Leitor } 2\end{array}$} & \multirow{2}{*}{ Total } \\
\hline & & $\begin{array}{l}\text { Ruim/Muito } \\
\text { ruim }\end{array}$ & Moderada & Bom/Excelente & \\
\hline \multirow{2}{*}{ Ruim/Muito ruim } & $\mathrm{n}$ & 0 & 1 & 0 & 1 \\
\hline & $\%$ & 0,0 & 1,5 & 0,0 & 1,5 \\
\hline \multirow{2}{*}{ Moderada } & $\mathrm{n}$ & 1 & 1 & 3 & 5 \\
\hline & $\%$ & 1,5 & 1,5 & 4,5 & 7,6 \\
\hline \multirow{2}{*}{ Bom/Excelente } & $\mathrm{n}$ & 1 & 4 & 55 & 60 \\
\hline & $\%$ & 1,5 & 6,1 & 83,3 & 90,9 \\
\hline \multirow{2}{*}{ Total } & $\mathrm{n}$ & 2 & 6 & 58 & 66 \\
\hline & $\%$ & 3,0 & 9,1 & 87,9 & 100,0 \\
\hline
\end{tabular}

Coeficiente de concordância de Gwet (IC 95\%): 0,83 (0,73; 0,94).

\section{Distorção da imagem - ADC}

\begin{tabular}{|c|c|c|c|c|c|}
\hline \multirow{2}{*}{\multicolumn{2}{|c|}{$\begin{array}{l}\text { Distorção da imagem - ADC IVIM } \\
\text { - Leitor } 1\end{array}$}} & \multicolumn{3}{|c|}{ Distorção da imagem - ADC IVIM - Leitor 2} & \multirow[b]{2}{*}{ Total } \\
\hline & & $\begin{array}{l}\text { Ruim/Muito } \\
\text { ruim }\end{array}$ & Moderada & Bom/Excelente & \\
\hline \multirow{2}{*}{ Ruim/Muito ruim } & $\mathrm{n}$ & 1 & 1 & 0 & 2 \\
\hline & $\%$ & 1,5 & 1,5 & 0,0 & 3,0 \\
\hline \multirow{2}{*}{ Moderada } & $\mathrm{n}$ & 1 & 6 & 5 & 12 \\
\hline & $\%$ & 1,5 & 9,1 & 7,6 & 18,2 \\
\hline \multirow{2}{*}{ Bom/Excelente } & $\mathrm{n}$ & 0 & 4 & 48 & 52 \\
\hline & $\%$ & 0,0 & 6,1 & 72,7 & 78,8 \\
\hline \multirow{2}{*}{ Total } & $\mathrm{n}$ & 2 & 11 & 53 & 66 \\
\hline & $\%$ & 3,0 & 16,7 & 80,3 & 100,0 \\
\hline
\end{tabular}

Coeficiente de concordância de Gwet (IC 95\%): 0,80 (0,68; 0,92).

\begin{tabular}{|c|c|c|c|c|c|}
\hline \multirow{2}{*}{\multicolumn{2}{|c|}{$\begin{array}{l}\text { Distorção da imagem - ADC } \\
\text { FOCUS - Leitor } 1\end{array}$}} & \multicolumn{3}{|c|}{ Distorção da imagem - ADC FOCUS - Leitor 2} & \multirow[b]{2}{*}{ Total } \\
\hline & & $\begin{array}{l}\text { Ruim/Muito } \\
\text { ruim }\end{array}$ & Moderada & Bom/Excelente & \\
\hline \multirow{2}{*}{ Ruim/Muito ruim } & $\mathrm{n}$ & 1 & 0 & 0 & 1 \\
\hline & $\%$ & 1,5 & 0,0 & 0,0 & 1,5 \\
\hline \multirow{2}{*}{ Moderada } & $\mathrm{n}$ & 1 & 4 & 3 & 8 \\
\hline & $\%$ & 1,5 & 6,1 & 4,5 & 12,1 \\
\hline \multirow{2}{*}{ Bom/Excelente } & $\mathrm{n}$ & 0 & 2 & 55 & 57 \\
\hline & $\%$ & 0,0 & 3,0 & 83,3 & 86,4 \\
\hline \multirow{2}{*}{ Total } & $\mathrm{n}$ & 2 & 6 & 58 & 66 \\
\hline & $\%$ & 3,0 & 9,1 & 87,9 & 100,0 \\
\hline
\end{tabular}

Coeficiente de concordância de Gwet (IC 95\%): 0,90 $(0,81 ; 0,98)$. 
Artefatos - ADC

\begin{tabular}{|c|c|c|c|c|c|}
\hline \multirow{2}{*}{\multicolumn{2}{|c|}{ Artefatos - ADC IVIM - Leitor 1}} & \multicolumn{3}{|c|}{ Artefatos - ADC IVIM - Leitor 2} & \multirow[b]{2}{*}{ Total } \\
\hline & & $\begin{array}{l}\text { Ruim/Muito } \\
\text { ruim }\end{array}$ & Moderada & Bom/Excelente & \\
\hline \multirow{2}{*}{ Ruim/Muito ruim } & $\mathrm{n}$ & 2 & 0 & 0 & 2 \\
\hline & $\%$ & 3,0 & 0,0 & 0,0 & 3,0 \\
\hline \multirow{2}{*}{ Moderada } & $\mathrm{n}$ & 2 & 5 & 1 & 8 \\
\hline & $\%$ & 3,0 & 7,6 & 1,5 & 12,1 \\
\hline \multirow{2}{*}{ Bom/Excelente } & $\mathrm{n}$ & 0 & 11 & 45 & 56 \\
\hline & $\%$ & 0,0 & 16,7 & 68,2 & 84,8 \\
\hline \multirow{2}{*}{ Total } & $\mathrm{n}$ & 4 & 16 & 46 & 66 \\
\hline & $\%$ & 6,1 & 24,2 & 69,7 & 100,0 \\
\hline
\end{tabular}

Coeficiente de concordância de Gwet (IC 95\%): 0,74 (0,61; 0,87).

\begin{tabular}{|c|c|c|c|c|c|}
\hline \multirow{2}{*}{\multicolumn{2}{|c|}{ Artefatos - ADC FOCUS - Leitor 1}} & \multicolumn{3}{|c|}{ Artefatos - ADC FOCUS - Leitor 2} & \multirow[b]{2}{*}{ Total } \\
\hline & & $\begin{array}{l}\text { Ruim/Muito } \\
\text { ruim }\end{array}$ & Moderada & Bom/Excelente & \\
\hline \multirow{2}{*}{ Ruim/Muito ruim } & $\mathrm{n}$ & 1 & 0 & 0 & 1 \\
\hline & $\%$ & 1,5 & 0,0 & 0,0 & 1,5 \\
\hline \multirow{2}{*}{ Moderada } & $\mathrm{n}$ & 3 & 2 & 2 & 7 \\
\hline & $\%$ & 4,5 & 3,0 & 3,0 & 10,6 \\
\hline \multirow{2}{*}{ Bom/Excelente } & $\mathrm{n}$ & 0 & 6 & 52 & 58 \\
\hline & $\%$ & 0,0 & 9,1 & 78,8 & 87,9 \\
\hline \multirow{2}{*}{ Total } & $\mathrm{n}$ & 4 & 8 & 54 & 66 \\
\hline & $\%$ & 6,1 & 12,1 & 81,8 & 100,0 \\
\hline
\end{tabular}

Coeficiente de concordância de Gwet (IC 95\%): 0,81 (0,69; 0,92). 